\title{
Propositions pour une typologie des méthodes de langues
}

Résumé de la thèse de doctorat d'État en Lettres et Sciences humaines soutenue par Henri Besse (juin 2000)

\section{Henri Besse}

\section{(2) OpenEdition}

Journals

Édition électronique

URL : https://journals.openedition.org/dhfles/2063

DOI : $10.4000 /$ dhfles.2063

ISSN : 2221-4038

Éditeur

Société Internationale pour l'Histoire du Français Langue Étrangère ou Seconde

Édition imprimée

Date de publication : 1 juin 2001

ISSN : 0992-7654

Référence électronique

Henri Besse, "Propositions pour une typologie des méthodes de langues », Documents pour l'histoire du français langue étrangère ou seconde [En ligne], 26 | 2001, mis en ligne le 04 septembre 2014, consulté le 27 mai 2021. URL : http://journals.openedition.org/dhfles/2063 ; DOI : https://doi.org/ $10.4000 /$ dhfles. 2063

Ce document a été généré automatiquement le 27 mai 2021.

(C) SIHFLES 


\section{Propositions pour une typologie des méthodes de langues ${ }^{1}$}

Résumé de la thèse de doctorat d'État en Lettres et Sciences humaines soutenue par Henri Besse (juin 2000)

\section{Henri Besse}

1 Cette thèse ${ }^{2}$ tend à préciser les notions et les raisonnements auxquels les didacticiens occidentaux des langues étrangères ou secondes (désormais L2) ont eu, et ont toujours, recours pour raisonner des " méthodes » destinées à les enseigner ou à les faire apprendre.

Une double interrogation l'oriente. Si les « méthodes » sont, comme l'estimait Stern (1983), « des combinaisons relativement fixes de croyances (a relatively fixed combination of language teaching beliefs) ", pourquoi ne pas étudier ces "croyances " en tant que telles, et pourquoi ne, pas étudier ces « combinaisons » que les didacticiens des L2 en proposent sous l'appellation de " méthodes », en s'interrogeant sur ce qu'elles présupposent ? En d'autres termes, il s'agit de reprendre, dans un domaine qui le pratique constamment sans toujours l'expliciter, le «syllogisme pratique " par quoi Aristote expliquait, à partir du désir et de la croyance qui la motivent, l'action humaine : «L'agent désire que $p$, et il croit que faire a est le meilleur moyen d'obtenir que $p$; par conséquent, il fait $a$, " Syllogisme qui revient à prêter une pertinence explicative à des états mentaux, tels « le fait de vouloir que $p$ » ou « le fait de croire que $p$ », états qui sont liés aux représentations, ordinaires ou plus savantes, dont on dispose quant à ce « $»$. Théorie de l'action, actuellement reprise par certains « cognitivistes », qui revient à poser que les représentations (celles qu'on se fait du but à atteindre et des moyens à même de le réaliser) peuvent être considérées comme la « cause » de l'action menée.

3 Ces représentations, fussent-elles réputées scientifiques, sont tributaires des traditions culturelles, en particulier scolaires ou universitaires, au sein desquelles elles sont transmises. Ce qui revient à dire que l'analyse de ces représentations n'est objectivable que si celui qui effectue cette analyse est à même de se déprendre ou de se décentrer quelque peu de la tradition dans laquelle il s'inscrit lui-même, à commencer par la langue dans laquelle il la mène. Et c'est, en grande partie, faute d'avoir eu accès à une 
documentation fiable sur des traditions non occidentales (indienne, arabe ou chinoise) que nous avons eu recours à l'histoire de la tradition occidentale, se décentrer dans le temps étant un moyen de pallier la difficulté qu'il y a encore, en didactique des $\mathrm{L}^{3}$, à se décentrer dans l'espace.

\section{Chapitre 1 : Questions de méthode(s)}

4 Ce chapitre s'efforce de clarifier les notions didactiques qui seront utilisées dans les investigations des chapitres suivants, et d'expliciter le point de vue et la démarche adoptés.

\subsection{Quelques notions méthodologiques}

5 Sont d'abord précisées les notions, constamment présentes dans les discours des didacticiens des L2 sans y être toujours clairement définies, de « langue maternelle » et de " seconde ou étrangère ", ainsi que celle de «transposition didactique » dont nous considérons qu'elle ne peut être appliquée à l'acte d'enseigner/apprendre une L2 comme elle peut l'être, par exemple, dans la didactique des mathématiques ou dans celle de la physique, ce qui explique la relative spécificité, à la fois historique et conceptuelle, de la didactique des L2. En d'autres termes, il s'agit de faire de notions (nous réservons le terme de concept à celles qui sont véritablement " construites " par une théorie) plus ou moins ordinaires ce que G. Bachelard appelle des " notions précisées ».

6 Une " langue » est définie - d'un point de vue qui emprunte à la fois à A. Meillet, $\mathrm{H}$. Weinrich, F. de Saussure, W. Labov, et quelques autres - comme un ensemble de variétés langagières à même de permettre, entre ceux qui en ont l'usage, une intercompréhension plus ou moins immédiate. Dans cet ensemble, sont distinguées les variétés dites vernaculaires (dialectes, sociolectes, technolectes, idiolectes) et la variété dite cultivée (celle qui est orthographiée, normalisée, codifiée par les dictionnaires, grammaires, rhétoriques, illustrée par des œuvres littéraires, et qui est généralement la variété enseignée), que nous dénommons par substantivation du qualificatif qui distingue une langue d'une autre (le français, l'allemand).

7 Chaque variété langagière y est conçue - d'un point de vue qui emprunte à $E$, Sapir, C. Lévi-Strauss et quelques autres - comme mettant enjeu des éléments non strictement linguistiques mais indispensables à ses usages appropriés. Nous parlons de culture pour ceux qui sont liés à l'usage d'une variété vernaculaire, et de Culture pour ceux qui le sont à la variété cultivée. Quant à la relation entre langue et culture ou Culture, nous optons pour une thèse qui n'est ni celle de Meillet soutenant que c'est la culture qui détermine la langue, ni celle qui affirme l'inverse (dite, souvent, « de Sapir-Whorf»).

Les notions de "langue maternelle/seconde/étrangère " s'inscrivent dans la très longue histoire des institutions scolaires. On peut y distinguer, selon que les langues y ont été ou non enseignées, au moins trois dispositifs terminologiques. Le plus ancien (du Moyen Age au début du XVIIe siècle) oppose les langues dites littéraires ou savantes, celles qui étaient étudiées et pratiquées dans les cercles relativement savants, aux langues dites vulgaires (qu'elles soient maternelles ou étrangères) qui n'étaient pas scolairement enseignées, bien qu'elles fussent parlées par le plus grand nombre (par le vulgaires). Le dispositif le plus stable (du XVIIIe siècle à la première moitié du XXe 
siècle) tend à opposer les langues mortes aux langues vivantes, lesquelles ne seront vraiment enseignées dans un cadre scolaire qu'au XIXe siècle. L'on aura alors tendance à les dénommer langues anciennes vs. modernes. Le dispositif le plus récent - qui ne date, dans son utilisation proprement didactique, que de la seconde moitié du XXe siècle oppose la langue maternelle aux langues étrangères (anciennes ou modernes, mortes ou vivantes). Ce qui fait alors opposition n'est plus le fait que la langue soit enseignée ou non, mais qu'elle l'est, en principe, différemment selon qu'elle est réputée maternelle ou non. Prise en compte, encore timide, d'un critère non « statutaire » ou non « officiel ", la " nature » de la langue enseignée pour chaque apprenant, critère auquel nous accordons la priorité en didactique des langues.

9 Ce qui nous permet de préciser ces notions à partir de trois questions hiérarchisées : $\mathrm{I}^{\circ}$ L'apprenant pratique-t-il déjà, ou non, au moins une des variétés de la langue enseignée ? $2^{\circ}$ Est-elle, ou non, langue d'enseignement d'autres " matières " scolaires qu'elle-même ? $3^{\circ}$ L'une ou l'autre de ses variétés est-elle, ou non, pratiquée dans l'environnement non scolaire de l'apprenant ? En raison de la priorité que nous accordons à la première (relative à la " nature » de la langue enseignée pour chaque apprenant) sur les deux autres (relatives au « statut » de cette langue dans l'institution scolaire ou hors de celle-ci), il est clair que les notions ainsi précisées de "langue maternelle/seconde/étrangère " ne sont vraiment pertinentes que dans les débuts de l'enseigner/apprendre, et quelles ne sont applicables qu'après enquête individualisée (il est toujours hasardeux d'inférer du « statut » d'une langue à sa « nature " pour tel ou tel apprenant).

10 Quant à la conception de l'objet enseigné/appris, en l'occurrence ici une L2, la tradition occidentale paraît avoir oscillé entre ce qu'on appelle, en français, un usage (" pratique que l'ancienneté ou la fréquence rend normale dans une société donnée ») et un savoir (« ensemble de connaissances plus ou moins systématiques, acquises par une activité mentale suivie »). Il s'ensuit un dilemme beaucoup plus prégnant pour les enseignants des L2 vivantes que pour ceux des L2 mortes. Afin de répondre à la demande sociale, les premiers se doivent de privilégier l'usage, oral ou écrit, des L2 vivantes qu'ils enseignent. Mais pour être considérés comme de véritables enseignants, comme ayant une compétence autre que celle de simples locuteurs se bornant à transmettre l'usage des langues qu'ils pratiquent (telles les nurses ou les natifs ignorants), ils doivent prouver que leur activité professionnelle engage des savoirs légitimés par l'Université dont ils relèvent.

11 L'ensemble de ces précisions notionnelles s'inscrit dans le cadre de ce que, vers 1970, on a recommencé - une " première didactique » a émergé en Allemagne au début du XVIIe siècle avant de passer en France au XVIIIe, pour y être à peu près complètement oubliée au XIXe et dans les deux premiers tiers du XXe siècle - à appeler la didactique des langues, entendue comme une réflexion ne dissociant pas l'étude de l'objet enseigné/ appris de la manière dont cet objet peut, ou doit être, scolairement transmis. Option qui n'est donc ni celle de la "linguistique appliquée ", quand la linguistique " structurale " était réputée apporter des réponses " scientifiques " au comment enseigner/apprendre les L2, ni celle des sciences de l'éducation telles qu'elles ont émergé à la fin du XIXe siècle et qui tendent à raisonner l'enseigner/apprendre sans trop tenir compte de la spécificité des objets enseignés/appris.

Sont ensuite successivement rappelées et critiquées une dizaine de propositions qui ont été avancées, entre 1960 et 1990, à propos des «méthodes » de L2 : celle de Anthony 
(1963) ; de Halliday, Mc Intosh, Strevens (1964) et de Mackey [1965 $]^{4}$; de b (1969), Dabène (1972) et du Dictionnaire de didactique des langues (Galisson \& Coste dir., 1976) ; de Richards \& Rodgers (1986) ; de Puren (1988) ; ainsi que trois critiques de la notion même de « méthode » (celles de Debyser, 1973 ; de Stern, 1983 ; et de Allwright, 1991). Propositions que nous confrontons aux nôtres (Besse, 1985), afin de préciser celles que nous avons retenues dans cette étude.

Elles consistent, pour l'essentiel, à distinguer soigneusement, dans les discours constitutifs de l'enseigner/apprendre les L2, trois niveaux d'analyse différents:

14 -Celui des discours qui traitent du comment enseigner/apprendre les L2, discours qui sont produits par des spécialistes de ce domaine s'adressant à d'autres spécialistes, et qui visent à expliciter, fonder ou rationaliser ce que nous appelons les méthodes et les approches ${ }^{5}$;

15 -Celui des discours qui mettent en œuvre ces « méthodes » ou " approches », pour une L2 donnée, dans des ensembles pédagogiques ou des manuels, discours qui sont produits par des spécialistes (les mêmes que ceux du niveau précédent ou d'autres) à destination d'un public potentiel d'apprenants de cette L2. Ces discours leur sont parfois adressés directement (manuel d'auto-apprentissage) mais, le plus souvent, indirectement, par le truchement des maitres qui utilisent un manuel.

16 - Celui des discours qui sont effectivement tenus dans une classe où l'on enseigne/ apprend une L2, que ce soit avec ou sans manuel. Ces discours, qui sont les seuls à réellement assurer l'enseignement/apprentissage d'une L2, sont infiniment plus complexes que ceux des deux niveaux précédents, et ne sont que partiellement déterminés par eux, même quand le maître y utilise à un manuel.

Ces trois niveaux d'analyse posés, nous précisons, terminologiquement, les notions auxquelles nous avons recours dans l'analyse de chacun d'eux.

18 Le premier implique donc ce que nous nommons les méthodes et les approches. Une " méthode » est définie comme un ensemble de (fragments de) discours qui relève de ce niveau et qui vise à promouvoir un certain point de vue et certaines hypothèses relatives aux langues et à leur appropriation guidée, en tant que L2, par des enseignants, ensemble qui vise à rationaliser leurs pratiques par une combinaison, plus ou moins cohérente, de techniques (d'enseignement) qui s'y trouvent dûment préconisées. Nous réservons le terme approche aux grands courants qui sont à même d'engager différentes méthodes, qui traversent ou divisent l'ensemble des discours constitutifs de la didactique des L2.

Le second correspond au niveau des manuels, c'est-à-dire aux ouvrages (livres ou ensembles pédagogiques plus complexes) où sont mises en œuvre, pour une L2 donnée et pour un public potentiel visé, les propositions d'une où plusieurs " méthodes ». Ces manuels constituent une sorte de "prêt-à-enseigner " pour les maîtres et une sorte de " prêt-à-apprendre » pointes élèves. Ils supposent des procédures d'élaboration quant à ce que nous appelons - nous démarquant quelque peu des propositions de Mackey (1965) - : la sélection des éléments de la L2 (d'une de ses variétés, en général la variété cultivée) qui ont été choisis pour être enseignés/appris en priorité ; la progression selon laquelle ces éléments sont regroupés par unité (groupages) ou échelonnés dans la suite des unités prévues par le manuel (séquences); la présentation ou type de discours selon lequel on y présente la L2 ; l' exploitation ou les exercices et activités qu'on y trouvent afin d'enseigner/apprendre le contenu de chaque unité ; enfin, dans certains manuels, 
les contrôles qui y sont proposés afin de vérifier ce qui a été appris. Chacune de ces procédures met en jeu, pour un manuel donné, des techniques, celles qui sont préconisées par sa (ou ses) méthode(s) de référence, mais qui ne sont, à ce niveau, qu'exempliflées.

Le troisième est celui des discours effectivement tenus dans une classe particulière où l'on commence à enseigner/apprendre une L2, qu'on y utilise ou non un manuel. Nous le réduisons - notre propos n'étant pas de l'étudier en tant que tel - aux sept " circonstances » que la rhétorique classique assigne à tout discours : QUI, QUOI, OÙ, PAR QUELS MOYENS (quibus auxiliis), POURQUOI, COMMENT (quomodo ou " la manière de l'exécution ») et QUAND. Deux notions de ce troisième niveau sont seules précisées celle des pratiques (d'une technique d'enseignement), par quoi nous entendons la manière particulière (quomodo) qu'a chaque enseignant de mettre en œuvre une technique préconisée par la méthode ou exemplifiée par le manuel. Et celle des procédés, par quoi nous entendons les démarches que le maître adopte de sa propre initiative, alors qu'elles ne sont prévues ni dans le manuel ni dans la méthode.

Enfin, pour marquer la coupure que nous instaurons entre les deux premiers types de discours et le troisième, nous nommons méthodologie l'étude systématique des discours constitutifs des méthodes et des manuels; et pédagogie, l'étude ou la pratique effective des discours constitutifs des classes de L2.

Il est clair que ce dispositif - dont les présupposés reviennent à admettre qu'une L2 est " enseignable » - n'est pas strictement déduit de quelques principes. Il s'agit d'un effort, quelque peu explicité, visant à rationaliser terminologiquement ce que certains didacticiens des L2, essentiellement d'expression française et anglaise, ont proposé, depuis une trentaine d'années, pour raisonner discursivement de ce domaine. Cette terminologie est d'ailleurs mise à l'épreuve dans notre propre discours méthodologique, afin de valider, empiriquement, son caractère opératoire, dans un métadiscours que nous disons à visée scientifique.

\subsection{Pour un point de vue et une méthode d'ordre historico- épistémologique}

Dans une seconde partie, nous nous efforçons de préciser le point de vue qui oriente nos analyses, lequel relève d'une anthropologie plus culturelle que physique, et la méthode que nous avons suivie, laquelle s'efforce à une certaine « clarté [...] obtenue de manière discursive [...] en faisant fonctionner les notions, en variant les exemples » (G. Bachelard). En d'autres termes, il s'agit de tenir un discours qui soit, autant que possible, non contradictoire et constamment éprouvé par l'analyse minutieuse de " cas " qui sont à même de l'infirmer ou de le confirmer. "Méthode des cas " qui doit beaucoup - modestie gardée - à Ch. S. Peirce et à T. S. Kuhn.

Pour typologiser les méthodes d'enseignement des L2, une démarche séduisante serait de les distinguer en fonction des processus d'apprentissage que chaque méthode est supposée provoquer chez les apprenants. Mais une telle démarche nous semble, dans l'état actuel de nos connaissances, prématurée.

En atteste notre critique du "modèle de Krashen ", dans ses versions successives qui vont de l'analyse de " l'enseignement formel » des L2 (Krashen \& Seliger, 1975) à « l'hypothèse de l'Acquisition/Apprentissage » (Krashen,1981,1989), en passant par celle 
$\mathrm{du}$ Moniteur. On sait que Krashen postule l'existence de deux « voies » pour s'approprier une L2, qu'il conçoit comme indépendantes, parce qu'on ne pourrait « passer de " l'une à l'autre (Learningdoes not 'turn into'acquisition). L'Acquisition serait privilégiée par les enfants, mais elle pourrait être aussi celle d'adolescents ou d'adultes qui s'approprient une L2 dans des " environnements informels ». Toutefois, chez ces derniers, l'Apprentissage l'emporterait en général, dans le cadre d'un « enseignement formel », ce qui entraverait leur Acquisition réussie de la L2.

D'où une " méthode ", The Natural Approach, qui vise à promouvoir, en classe de L2, la " voie » de l'Acquisition sur celle de l'Apprentissage. Méthode que Krashen \& Terrell (1983) théorise en cinq hypothèses, dont les quatre dernières explicitent ce que présuppose la première : 1) celle de l'Acquisition/Apprentissage (dont il vient d'être question) ; 2) celle, étroitement liée au "dispositif inné d'acquisition " que Chomsky postule chez tout être humain, d'un "Ordre naturel » faisant que les " morphèmes " d'une langue seraient toujours « acquis » dans le même ordre, quel que soit celui dans lequel ils se présentent à l'apprenant ; 3) celle du Comprehensible input, qui revient à alimenter ce dispositif " grammaticalisant " par des données qui lui soient compréhensibles et suffisamment riches pour l'inciter, par la compréhension qu'il en a, à progresser ; 4) celle du " Moniteur ", une sorte d'organe mental qui n'atteindrait son plein développement qu'à la puberté et dont la fonction serait de "surveiller » ou de " contrôler " les productions quand certaines conditions sont remplies (avoir le temps nécessaire, prêter attention à la forme, disposer d'une règle antérieurement apprise à laquelle on peut se référer) ; 5) celle du "Filtre affectif " enfin, qui serait liée aux attitudes des apprenants par rapport à la L2 et à l'enseignement, et qui renforcerait, chez les adolescents et les adultes, le rôle du Moniteur.

Le « modèle de Krashen » a été beaucoup critiqué, entre autres par Me Laughlin (1978, 1987), Bialystok (1981), Bibeau (1983), Besse (1984), Gregg (1984), Odlin (1986). On ne saurait dire qu'il en constitue une véritable théorie, par manque de cohérence à la fois interne (entre les cinq hypothèses) et externe (par rapport à ce qu'on sait par ailleurs).

Son indéniable succès, chez les didacticiens et les enseignants de L2, nous parait lié à ce qu'il permet de " parler de » croyances plus ou moins traditionnelles dans l'enseignement occidental des L2, en des termes évoquant ce qui a été leur préoccupation première, au moins durant la décennie 1975-1985, " ce-qui-peut-sepasser " chez l'apprenant. En d'autres termes, l'opposition Acquisition vs. Apprentissage permet de reformuler en termes « modernes » ce qui oppose, depuis des siècles en Occident, les tenants d'un enseignement des L2 "par usage ", plus ou moins calqué sur la manière dont on suppose que l'on acquiert "naturellement » une langue, aux tenants de leur enseignement " par règles ", qui prennent davantage modèle sur l'étude savante des langues.

Notre étude postule, à l'opposé du « modèle de Krashen, que l'organisation neurobiologique, qui rend tout être humain apte à s'approprier une langue (Ll ou L2), est loin de déterminer cette appropriation dans toutes ses composantes, en particulier celles sur lesquelles on peut didactiquement agir. Point de vue qui rejoint celui de Odlin (1986) ou de Giordan \& de Vecchi (1987), et qu'on peut qualifier d'« historico-naturel ». Il revient à poser que les méthodes d'enseignement des L2 sont des représentations destinées à apporter des réponses d'ordre pratique à certaines "situations-problèmes " caractéristiques de renseignement/apprentissage des L2. Ces représentations sont véhiculées par le discours des méthodes qui les préconise et le discours des manuels qui 
les exemplifie, et elles sont sous-tendues par ce que Giordan \& de Vecchi appellent des " conceptions » ou des "structures cognitives ", généralement léguées par la tradition didactique tout en présentant parfois des caractères novateurs, et que nous appelons des (pré)conceptions.

\section{Chapitre 2 : Inventorier et caractériser les méthodes}

Ce chapitre rappelle et critique d'abord douze « inventaires des méthodes » qui ont été proposés, entre 1965 et 1989, par des didacticiens des L2 d'expression française et anglo-américaine ; ensuite, quelques démarches avancées par les mêmes ou par d'autres, afin de caractériser, de manière plus systématique, ces « méthodes ».

Cet « état de l'art » nous conduit à préciser les critères typologiques que nous adoptons. Ils sont réductibles à quatre questions dont les réponses sont fortement tributaires du fait que l'on opte - quand ce choix demeure possible - ou bien pour un enseignement de la L2 au moyen de la Ll des élèves, ou bien pour un enseignement se privant de celle-ci. Questions et réponses qui ont donné lieu, au moins depuis le XIXe siècle, à des débats et polémiques révélateurs des (pré)conceptions et points de vue sous-jacents, tant il est vrai qu'on ne débat que de ce qui fait problème, que de ce qui pose question.

\subsection{Inventorier les méthodes}

Les « inventaires de méthodes " présupposent l'existence de ces dernières comme s'il s'agissait de réalités objectivement distinctes qu'il suffirait d'énumérer et de caractériser plus ou moins systématiquement. Il se borne, le plus souvent, à de simples relevés - à partir d'un repérage de leurs dénominations - des " méthodes » et des représentations qui leur sont associées, telles qu'elles sont véhiculées dans les discours constitutifs de la didactique des L2.

L'« inventaire » que propose Mackey [1965], ne respecte pas strictement la distinction « méthode/manuel » telle que précisée ci-dessus. Il comprend, très brièvement caractérisées, dix-sept méthodes principales, auxquelles il faudrait ajouter des " variantes moins répandues » et " les centaines de méthodes désignées par le nom de leurs auteurs ». Les caractérisations qu'il en fait esquissent un regroupement de sept ou huit d'entre elles autour de la méthode directe $e^{6}$ (priorité à l'oral, pas de traduction magistrale, grammaire enseignée " en situation »), et de six ou sept autres autour de la méthode grammaire-traduction (priorité à l'écrit, traduction par le maître et par les élèves, grammaire " expliquée »). Quatre méthodes enfin y sont vues, dans les caractérisations qui en sont faites, comme étant intermédiaires à ces deux regroupements. En d'autres termes, la méthode directe et la méthode grammairetraduction constituent, aux yeux de Mackey, des sortes d'archétypes à même d'orienter la réflexion méthodologique.

Dans un guide pédagogique qui a connu un grand succès en Amérique du Nord Rivers [1968] décrit des classes de L2, où le maître est supposé appliquer quatre méthodes différentes (grammaire-traduction, directe, par la lecture, audio-orale); auxquelles elle en ajoute une cinquième, la méthode éclectique qui adopterait « les meilleures techniques de toutes les méthodes » et qui serait un compromis " imaginatif, énergique et avide d'expérience » entre méthodes grammaire-traduction et directe. 

audio-orale, audio-visuelle). Mais, dans un article de 1985, il assimile plus ou moins méthode audio-orale et méthode audio-visuelle, et leur adjoint " les approches dites fonctionnelles, notionnelles et communicatives ".

\& Di Pietro (1970) cherchent, quant à eux, à « dépasser la division des méthodes ». Ils en proposent une caractérisation plus systématique fondée d'une part sur le fait que la « méthode » est, ou n'est pas, « orientée-vers-les-règles », et d'autre part sur la (pré)conception ou « universelle » ou « immanente à chaque langue » qu'on s'y fait de ces règles. Ce qui revient, selon les croyances de l'époque, à opposer les méthodes qui s'appuient sur la grammaire traditionnelle à celles qui " appliquent " une grammaire structurale alors dominante. Ils distinguent donc trois "stratégies de l'enseignement " des L2, qui sont dites grammaire-traduction, directe et audio-orale.

Le Dictionnaire de didactique des langues (Galisson \& Coste, 1976) propose implicitement on le rétablit aisément à partir des différentes entrées - un inventaire de cinq ou six méthodes, où la méthode grammaire-traduction n'apparaît que dans les caractérisations des méthodes disposant d'une entrée, en particulier dans celle réservée à la méthode directe. Par rapport aux inventaires précédents, ce dictionnaire se singularise par le fait que la méthode audio-visuelle y est subdivisée en méthode intégrée et en méthode structuro-globale, et par le fait qu'on y retient aussi la méthode cognitive et les « méthodes actives ».

Stern (1983) - qui conteste la notion de "méthode » en ce qu'elle ne serait pas « une base suffisante pour conceptualiser l'enseignement des L2 "-n'en consacre pas moins tout un chapitre à décrire systématiquement (histoire, objectifs, techniques préconisées, références théoriques, évaluation) six méthodes (grammaire-traduction, directe, audio-orale, audio-visuelle, The Reading Method et The Cognitive Theory), auxquelles il ajoute, en note, la méthode Silent Way, la Suggestopédie, la Dartmouth Method et The Natural Approach de Terrell (avant donc que Krashen ne l'aide à la théoriser).

39 A ces six méthodes, quatre autres inventaires nord-américains, (Hammerly, 1985 ; Larsen-Freeman, 1986 ; Richards \& Rodgers, 1986 ; Germain, 1989 et 1993) ajoutent la méthode communautaire (Community Language Learning), la méthode physique (The Total Physical Response), et quelques autres, dont la méthode situationnelle, absente des autres inventaires bien qu'elle soit antérieure à la méthode audio-orale (Mackey se borne à signaler son appellation).

40 Enfin, nous avons retenu deux autres « inventaires » européens, celui de Puren (1988) et celui de Marion (1988), parce qu'ils diffèrent des précédents par le mode de caractérisation adopté.

41 Puren s'appuie, dans une perspective nettement diachronique, sur la notion de " méthodologies ", par quoi il entend des ensembles « cohérent de procédés, techniques et méthodes qui (se sont)révélé(s) capable(s), sur une certaine période historique et chez des concepteurs différents, de générer des cours relativement originaux par rapport aux cours antérieurs et équivalents entre eux quant aux pratiques d'enseignement /apprentissage induites ". Ce qui lui permet de périodiser l'enseignement des L2 vivantes en France, du XIXe siècle à nos jours, en quatre " méthodologies » : 
42

d'apprentissage " propre, lequel pourrait ou non convenir aux élèves selon leur « style d'apprentissage ». Propositions qui, tout comme celles de Krashen, nous paraissent beaucoup spéculer sur « ce-qui-se-passe-dans-l'esprit » de l'apprenant, et qui vont directement à rencontre de celles que nous avons avancées en conclusion de notre premier chapitre. dans un tableau récapitulatif permet de constater que seules deux méthodes (grammaire-traduction et directe) sont prises en compte par tous. Ce qui, de nouveau, atteste de la prégnance, dans les discours méthodologiques de la fin du XXe siècle, des archétypes sous-jacents à l'inventaire de Mackey. Aucun d'entre eux n'est exempt d'incohérences internes, liées à ce qu'on s'y tient trop souvent aux appellations attestées dans les discours des méthodes/manuels et aux justifications théorisantes avancées, en négligeant quelque peu l'analyse des techniques (d'enseignement) qui y sont préconisées ou exemplifiées.

54 Toute typologie rationnellement construite implique une reconstruction abstraite des données sur lesquelles elle porte, et cette reconstruction se doit d'être fondée sur un nombre limité de critères, dûment et préalablement, explicités. Les techniques d'enseignement, telles que nous les entendons, nous semblent les mieux à même d'être posées comme des invariants typologiques de ce type, en ce qu'elles sont aisément repérables tant dans le discours des méthodes que dans celui des manuels, et en ce 
qu'elles présentent une certaine inertie, à la fois diachronique et synchronique, par rapport aux théories dont on se réclame.

\subsection{Caractériser les méthodes}

Des douze « inventaires » retenus, seuls six caractérisent un peu systématiquement - à partir d'un modèle explicité - les méthodes qu'ils répertorient.

Bosco \& Di Pietro spécifient leurs trois "stratégies d'enseignement " à l'aide d'une analyse en " traits distinctifs ", inspirée des démarches structurales de type componentiel. Chaque " trait » y est conçu comme binaire (+ vs. -), mais la présence d'un « trait " n'implique pas toujours l'absence de son opposé. " Huit traits psychologiques » sont retenus, qui sont plus ou moins clairement empruntés à diverses théories psychologiques ou éducatives. Et «trois traits linguistiques " qui renvoie à l'opposition - telle qu'elle était admise dans les années soixante - entre grammaire traditionnelle et linguistique structurale. Modèle qui nous paraît peu cohérent (les " traits " se recoupent ; ils ne sont pas hiérarchisés ; ils supposent une interprétation intuitive des données), sans doute parce qu'il relève d'un point de vue par trop " linguistique appliquée » ou «psychologie appliquée ».

Krashen \& Seliger caractérisent « l'enseignement formel » des L2 selon un modèle plus strictement méthodologique, qui reposent sur huit " traits ", dont deux seraient " universels » (en ce qu'ils seraient toujours présents : + discrete point et + feedback) et six « locaux » (en ce qu'ils dépendraient des méthodes : \pm deductive, \pm explicit, \pm sequence, focus on vs. focus away...). "Traits " dont il est parfois difficile de savoir à quel niveau d'analyse (méthode/manuel/classe) ils renvoient.

Hammerly propose "une théorie intégrée » de l'enseignement des L2, théorie pluridisciplinaire fondée sur une cinquantaine de "principes » (sans compter leurs corollaires), dont une douzaine empruntés à la linguistique (structurale et pragmatique), une quinzaine à la psychologie (d'orientation plutôt cognitiviste), une autre quinzaine aux sciences de l'éducation, et quelques autres à diverses « sciences " connexes (psychométrie, sociolinguistique, technologie...). Les quinze " principes » plus ou moins empruntés aux sciences de l'éducation (Teaching Principles) sont les seuls à vraiment porter sur l'enseignement des L2, mais ils ne sont pas assez neutres et généraux pour être intégrés tels quels dans une méthodologie des méthodes et manuels de L2, pré-dessinant par trop la méthode (The New CA-OB Method) que Hammerry cherche à promouvoir.

Pour Larsen-Freeman, toute classe de L2 répond d'une manière ou d'une autre, à dix questions formulées en termes de " principes » ou d'activités transposables à d'autres classes. Ces questions nous semblent plus aisément applicables aux classes mêmes qu'aux manuels ou aux méthodes telles que nous les entendons, les réponses dépendant de ce que Galisson et Puren appellent la "pédagogie induite ", avec les incertitudes que cette notion entraîne.

Richards \& Rodgers ne retiennent que six « composantes » : les objectifs, le contenu et la progression, les types d'activités, les rôles de l'apprenant, les rôles de l'enseignant, et le rôle du matériel pédagogique. Ce modèle nous parait, tout comme les précédents, souffrir d'un point de vue par trop "linguistique/psychologie appliquées " à un domaine qui n'est que marginalement celui de ces deux sciences. Mais il comprend des distinctions intéressantes quant aux objectifs (selon qu'ils sont orientés sur les 
processus d'apprentissage ou sur les « produits » résultant de celui-ci) et aux contenus (selon qu'ils sont centrés sur ces processus ou sur la matière à enseigner), même si ces distinctions sont critiquables dans les formulations proposées.

61 Germain élabore " un cadre conceptuel pour la didactique des langues » qui tente d'intégrer, au « modèle éducationnel » de Legendre (1988), une partie des propositions de Larsen-Freeman, Richards \& Rodgers, Besse (1985). Ce modèle réduit 1'« apprentissage ", quelle que soit la " matière » concernée, à un ensemble de relations biunivoques entre un milieu (M), un sujet (S), un objet (O), et un agent (A). Ce qu'il résume par la formule : $\mathrm{APP}=\mathrm{f}(\mathrm{S}, 0, \mathrm{M}, \mathrm{A})$, qui aurait " un caractère véritablement opératoire pour l'analyse des méthodes", pour peu qu'elle soit spécifiée : $\mathrm{S}$ renvoie à la conception de l'apprentissage et au rôle assigné à l'apprenant ; O réfère à la conception de la langue et de la culture ; A s'entend comme la conception du rôle de l'enseignant et du matériel pédagogique ; et $\mathrm{M}$ est relatif aux infrastructures, pédagogique et administrative, dans lesquelles s'inscrit inévitablement « la relation pédagogique » S-A0 . Ce modèle, ainsi spécifié, possède un pouvoir typologique quant ce que Germain appelle les " grands courants ", lesquels correspondent - même s'ils sont déterminés par un point de vue différent du nôtre - à ce que nous appelons les "approches". Germain en retient trois : un "courant linguistique " regroupant les méthodes " centrées sur la nature de la langue ", un " courant psychologique » regroupant celles qui sont " centrées sur l'apprentissage ", et un " courant intégré " regroupant celles qui sont « centrées sur la nature de la langue et sur l'apprentissage (processus et/ou conditions) ». C'est dans ce dernier qu'il range la méthode des séries de Gouin, mais pas la méthode directe, considérant qu'elle relève du " courant psychologique ». Modèle qui nous semble privilégier les références théoriques du discours des méthodes au détriment des objectifs ou finalités poursuivis, et qui se heurte à des difficultés quant aux trois niveaux d'analyse que nous avons distingués.

Stern ne propose pas un modèle, mais un repérage des « lieux » (au sens rhétorique) ayant suscité débats et polémiques depuis un demi-siècle, lesquels seraient réductibles à « trois problèmes majeurs »:

63 -The L1-L2 connection, c'est-à-dire les rapports qu'entretiennent la Ll et la L2 dans l'enseignement/apprentissage de cette dernière ;

64 -The explicit-implicit option, c'est-à-dire opter pour un enseignement explicite ou implicite des règles (grammaticales);

65 -The code-communication dilemma, ou alternative entre un enseignement centré sur le code de la L2 ou un enseignement centré sur les conditions de sa communication.

66 A ces trois « lieux » de désaccord qu'il considère comme « majeurs », Stern en ajoute un quatrième relatif aux progressions d'enseignement adoptées (faut-il, en particulier, enseigner simultanément les quatre habiletés ou les « décaler » temporellement?).

Quatre "lieux " qui sont effectivement présents dans tous les inventaires ou caractérisations présentés ci-dessus. Mais Stern nous paraît les formuler en des termes qui renvoient plus aux polémiques théorisantes qu'à des critères vraiment opératoires quant à la distinction des méthodes.

68 Écartant, en tant que critères typologiques possible, les procédures d'élaboration des manuels et les finalités poursuivies - qui doivent néanmoins être prises en compte, afin d'éviter de "se réfugier derrière le paravent des techniques et des procédés sur lesquels s'appuie empiriquement l'action éducative » (B. Jolibert, 1987) -, nous retenons 
finalement les « lieux » repérés par Stern comme étant les mieux à même de tenir compte des problèmes dont il faut nécessairement résoudre quand on commence à enseigner une L2. Faut-il encore que ces « lieux » soient reformulés en termes moins théorisants et plus strictement méthodologiques. On peut observer qu'ils mettent enjeu au moins quatre alternatives : 

et non par nécessité, les réponses apportées à notre première question font appel à des procédés de traduction interlinguale qui ont été raisonnes dès l'Antiquité (par Cicéron ou saint Jérôme), mais qui restent encore peu théorisés en dépit du développement récent de la " traductologie ». Ce qui frappe dans leur évolution bimillénaire, tels qu'on peut les observer dans les manuels de L2 au moins depuis le IIIe siècle jusqu'à nos jours, c'est qu'en ont été peu à peu éliminés les deux procédés sur lesquels Cicéron et saint Jérôme focalisaient leur réflexion (à savoir le mot à mot et la libre adaptation en langue-cible), pour s'en tenir à une voie moyenne, celle d'une " fidélité » qui facilite certes les évaluations scolaires ou universitaires, mais dont on voit mal à quoi elle peut correspondre dans les débuts de l'enseignememt/appentissage d'une L2. Et quand ces réponses ne recourent pas, toujours par méthode, à la L1 des élèves, elles mettent en jeu la relation des " mots " aux " choses " à même d'être dénotées par eux, relation débattue également depuis l'Antiquité (dans le Cratyle ou saint Augustin) et que les théories linguistiques ou sémiotiques modernes (entre autres celles de Saussure et de Peirce) n'ont pas totalement éclaircie.

\subsection{De l'enseignement du sens des signes de la L2} apportées au premier problème de Stern, tel que nous l'avons reformulé à la fin du précédent chapitre, et de préciser les notions auxquelles, dans ces réponses, on a nécessairement recours. 
-les «procédés figuratifs » qui assurent « la plupart des fonctions sémantiques » des précédents, «à l'exception du rapport dans le temps et l'espace du sujet parlant avec son entourage réel »;

-les "procédés contextuels ", dans lesquels on fait appel au vocabulaire de la L2 qui est déjà connu des élèves pour leur enseigner « la signification des mots nouveaux ou de nouvelles structures ».

Autant de distinctions qui nous semblent reposer sur des critères hétérogènes.

Kelly (1969), qui ne se réfère pas à Mackey, retient lui aussi quatre " traitements » du sens : « les équivalents en L1 »; les « gestes et objets »; les images; les « explications en L2 ». Les trois derniers l'auraient, au cours de l'histoire, emporté sur le premier.

Jakobson (1963) - qui récuse la tradition logique voulant que le sens d'un mot ou d'une proposition dépendent d'abord de la perception ou de l'expérience que l'on peut avoir de son dénoté, et qui reprend à sa manière Peirce et Saussure ( « il n'y a pas de signifié sans signe ») - réduit le problème du sens à trois sortes de traduction : « intralinguale ou reformulation »; « interlinguale ou traduction proprement dite »; « intersémiotique ou transmutation».

94 Ces propositions, souvent reprises en didactique des L2, nous y apparaissent comme relativement peu pertinentes et conduisent à des raisonnements approximatifs. Ainsi un élève qui commence à apprendre une $\mathrm{L} 2$ ne dispose pas du minimum de bilinguisme qui lui permettrait de réellement traduire ; tout au plus peut-il « surcoder » les signes de sa L1 au moyen de ce qu'il perçoit des signifiants de la L2. Mackey et Kelly, autre exemple, font remonter l'emploi systématique des images dans l'enseignement des L2 à l'Orbis Sensualium Pictus de Comenius, voyant en lui un précurseur des méthodes directe ou audio-visuelle, alors que les images de ce manuel sont constamment légendées au moyen d'équivalences L2-L1. D'où des caractérisations des techniques (d'enseignement) souvent imprécises.

95 C'est pourquoi nous avons entrepris la description quasi clinique d'une seule de ces techniques, qui est aisément repérable dans le discours des manuels et qui a été, sur la longue durée, théorisée dans le discours des méthodes de points de vue différents. Il s'agit de la traduction interlinéaire telle qu'elle a été utilisée en didactique des L2, au moins depuis le XVIe siècle jusqu'à nos jours, même si elle n'a guère été théorisée avant le XVIIIe siècle. Sont successivement analysées les propositions, à ce sujet, de J. Locke, de C. Chesneau Du Marsais, de C. F. Lizarde de Radonvilliers, de T. Robertson et de quelques autres. Cet essai de description atteste de la remarquable, même si elle n'est pas totale, inertie de cette technique, au cours des siècles, par rapport aux diverses théorisations qui en ont été proposées.

Ayant ainsi précisé la notion de "technique d'enseignement d'une L2 ", il nous a semblé plus cohérent - avec ce que nous avons précédemment avancé - de distinguer deux grands types de techniques d'enseignement $d u$ « sens étranger » : les techniques bilingues qui font appel à la traduction magistrale (effectuée par le manuel ou par le maitre), et les techniques monolingues qui récusent cette traduction magistrale et qui sont supposées enseigner « directement » ce sens, sans que le manuel ou le maître ait recours à la L1 des élèves.

7 La notion de " sens étranger » présuppose que ce sens n'échappe pas à la détermination de la L2 qui le manifeste. Mais pour que l'on puisse traduire, il faut nécessairement supposer qu'il y a «quelque chose » à défaut de la « même chose » qui le permette et 
qui, partant, échappe à la détermination des deux langues engagées dans cette opération. Le sens que nous disons " étranger » ne l'est que pour celui qui ignore la langue dans laquelle il est véhiculé, en l'occurrence pour l'élève débutant qui ne perçoit encore de la L2 que sa matérialité (sonore ou graphique) mais qui, sachant qu'elle est signifiante, cherche à appréhender ce " sens " à partir de ce qu'il connaît déjà (entre autres, sa L1). Ce " quelque chose " de commun qui rend possible la traduction nous paraît s'inscrire dans un continuum allant du sens réputé " propre " ou " premier ", assigné plutôt aux mots, aux sens tributaires des contextes et situations dans lesquels les énoncés qui les suscitent apparaissent, en passant par les sens réputés « figurés » ou " idiomatiques ", qu'ils soient stabilisés en langue ou qu'ils ne soient que des « effets de discours ». Pour plus de clarté discursive, nous réservons toutefois le terme de sens au " quelque chose » qui permet, en particulier dans les dictionnaires bilingues, d'établir des équivalences relativement stables et récurrentes entre langues, alors que le terme de signification l'est au "quelque chose », qu'il s'agisse de l'intenté ou de l'effet produit, que préservent les équivalences interprétatives, beaucoup moins stables parce que liées aux aléas des discours.

\subsection{Caractériser les techniques d'enseignement du « sens étranger}

) 
variable selon leurs mises en œuvre, leur propre diérèse, certaines usant de bulles iconisées, d'autres s'en tenant à la seule représentation des circonstances et des comportements jugés pertinents pour aider les élèves à comprendre les énoncés en L2.

Enfin, celui des techniques que nous disons de reformulation, telles qu'elles ont été surtout mises enjeu par les méthodes directe et audio-visuelle.

\subsection{Des préconceptions du « sens étranger»}


dans les pays de langue allemande, qu'au XVIIIe siècle. C'est dans cette tradition que s'inscrivent les caractérisations de ce que nous appelons la " traduction didactique " (théorisée, entre autres, par J.-P. Vinay \& J. Darbelnet, J.-R. Ladmiral ou E. Lavault), la « traduction pragmatique » (théorisée par V. van Osman Quine, G. Mounin, J.-C Catford D. Séleskovitch \& M.Lederer, M. Pergnier, ou J. Delisle), et la «traduction poétique » (telle que théorisée, dans la lignée d'Hölderlin ou de Goethe, par W. Benjamin ou H. Meschonnic).

Les querelles de la traduction n'étant « qu'une forme atténuée de la querelle du langage dans son ensemble » (G. Steiner), elles aident à discerner dans les représentations du langage et des langues, qu'elles soient ordinaires ou savantes, trois polarités. La première (pré)conçoit les langues comme des nomenclatures (d'ordre lexical plus que grammatical) d'un monde, perçu par les sens ou pensé, comme partagé par tous, sorte de « langage des choses » que cette (pré)conception pose comme étant plus ou moins universel. La seconde (pré)conçoit les langues comme étant à même d'imposer à ce perçu ou à ce pensé des configurations sémantiques qui leur seraient propres, et qui feraient qu'elles sont difficilement traduisibles. La troisième, enfin, les (pré)conçoit comme des activités sociales parmi d'autres, dont l'efficience dépend moins des signes employés que des environnements (contextes, situations) dans lesquels on les emploie.

110 Trois polarités où se retrouve quelque chose de la division médiévale du trivium (en logique, grammaire et rhétorique), ou de celle d'un Morris qui distingue, après Peirce, trois points de vue à partir desquels il est possible de théoriser le phénomène langagier (sémantique, syntactique, pragmatique). Trois polarités qui impliquent aussi des (pré)conceptions du signe linguistique différentes : si la théorisation qu'en propose Saussure permet de contester la (pré)conception inhérente aux nomenclatures et d'interpréter celle sous-jacente aux configurations sémantiques propres à chaque langue, elle nous paraît moins à même de rendre compte de ces polarités que la conception ternaire qu'en avance Peirce.

\section{Chapitre 4 : Enseigner la « grammaire » d'une langue étrangère}

111 Les réponses qui correspondent à notre seconde question sont les seules qui mettent en jeu une véritable "science "- du moins reconnue comme telle depuis l'époque hellénistique, lorsqu'elle s'est émancipée, pour des raisons ayant précisément à voir avec l'enseignement des langues, des spéculations proprement philosophiques. Il s'agit de cet ars grammatica qui ne deviendra la linguistique qu'au début du XIXe siècle, science dont le développement n'est pas dissociable des deux autres sciences constitutives du trivium médiéval, la logique et la rhétorique.

\subsection{Sur la notion de " grammaire » en didactique des $L 2$}

112 Au-delà de cette évidence que l'approche bilingue permet d'emblée de donner des explications grammaticales en L1 sur la L2, alors que l'approche monolingue ne le permet pas, l'option « explicite vs. implicite » (Stern) est déterminée par les finalités poursuivies (une finalité formative privilégie davantage les explications qu'une finalité utilitaire). Mais si débats et polémiques quant à l'enseignement de la " grammaire » d'une L2 perdurent depuis des siècles, c'est moins en raison de cette évidence que des 
ambiguïtés et incertitudes de la notion même de " grammaire ", et des (pré)conceptions de la « règle grammaticale » qui s'y trouvent engagées. C'est donc sur cette notion que nous revenons d'abord, à partir de quelques enquêtes sur les représentions qu'on s'en fait plus ou moins ordinairement.

En France, chez des étudiants de lettres (Combettes \& Lagarde, 1982) et chez des élèves $\mathrm{du}$ secondaire (Foerster, 1993), ces représentations divergent sensiblement. Les premiers, dans leur majorité, y voient soit Un « ensemble de règles ", soit « l'étude méthodique » de celles-ci, ce qui présuppose qu'ils admettent comme évidente une certaine rationalité des langues, à même d'en déterminer au moins le fonctionnement interne. D'ailleurs, cette majorité « s'étonne » qu'on puisse dire qu'il existe des langues qui n'ont pas de grammaire. Mais une minorité résiste à cette représentation, pourtant véhiculée par toute l'institution scolaire et universitaire française, et «s'étonne » à son tour qu'on puisse dire que l'enfant, quand il arrive à l'école, connaît sa grammaire. Elle voit dans la « grammaire » d'abord quelque chose qui s'apprend scolairement à des fins de correction, certains l'estimant "utile ", d'autres " peu utile et dure à apprendre ". Représentations contradictoires qui se retrouvent dans d'autres contextes éducatifs.

114 Des adultes nord-américains (Hayne \& Yorio, 1982), au passé polyglotte et apprenant une L2 à des fins non-utilitaires, jugent, dans leur majorité, que « l'explication des points de grammaire est 'importante' », mais une petite minorité maintient que ce n'est pas le cas. Enseignants et élèves allemands, de français ou d'anglais L2, s'avèrent tout aussi divisés (Zimmermann, 1984) : les enseignants se partagent, à peu près par moitié, entre ceux qui estiment que la « correction grammaticale » est importante et ceux pour qui elle ne l'est guère, même si tous consacrent beaucoup de leur temps de classe à la " grammaire » (70\% d'entre eux en partant d'exemples susceptibles d'aider les élèves à " découvrir par eux-mêmes » les règles ; $3 \%$ seulement les explicitant avant de les faire appliquer) ; les élèves, que le maitre procède inductivement ou déductivement, s'avèrent quelque peu dubitatifs ( $20 \%$ disent apprendre les règles par cœur, faute de les comprendre).

115 Dans les contextes enfin, où la tradition « grammaticalisante » est allogène, comme en Chine (Kuang-Jone, 1992), l'on observe, au moins dans les manuels de Ll, « une présence infime de métalangage grammatical ».

116 Les représentations ordinaires de ce qu'est la « grammaire » d'une langue apparaissent ainsi comme étant d'abord véhiculées par certaines traditions éducatives, dans lesquelles ces représentations sont moins unanimement partagées que celles afférentes au sens ou à la signification.

117 Il n'en va guère différemment pour les représentations plus savantes. Dictionnaires spécialisés (Dubois, 1973 et 1994 ; Portier, 1973 ; Mounin, 1974 ; Galisson \& Coste., 1976) ou études (Combettes \& Lagarde, 1982) distinguent de quatre à sept différentes acceptions du mot grammaire, dont certaines sont réputées "scientifiques ", réputées plus descriptives ou objectives que d'autres, qui ne seraient pas, "scientifiques", réputées normatives ou « scolaires ». Combettes \& Lagarde font observer que, pour être largement reçue, cette opposition entre grammaire "scientifique » et grammaire " scolaire " ne se retrouve guère dans les sciences de la nature, où il n'est guère question de physique ou de chimie "normatives, pédagogiques ou scolaires ». Et si certains spécialistes admettent que les descriptions grammaticales dépendent des théories qui les informent, aucun ne spécifie que grammaire prend une acception particulière dans grammaire structurale ou grammaire générative. 
118 Ainsi, s'il existe un certain consensus sur la « grammaire » en tant qu'artefact, celui-ci ne s'étend ni à son objet, c'est-à-dire au réel engagé par cet artefact, ni aux présupposés théoriques qui le constituent en tant que tel. Ce qui nous paraît lié à des incertitudes épistémologiques, qui nous conduisent à admettre une thèse voisine de celle de Milner (1989) : " une langue ou un usage ont un fonctionnement; le grammairien peut le décrire sous forme de règles; toutefois rien ne dit que ces règles soient davantage qu'une convention adoptée par le seul grammairien ». Proposition qui n'est pas contradictoire avec le fait que cette " convention ", pour peu qu'elle soit partagée et scolairement transmise, puisse fonctionner comme une norme de cette langue ou de cet usage, ou puisse en dire quelque chose qui soit de l'ordre du réel.

Thèse qui relève d'un réalisme modéré, et qui nous conduit à poser l'existence de trois types de connaissance grammaticale, dont le premier, inhérent à la pratique d'au moins une langue (proche du factum grammaticae de Milner) peut s'acquérir et subsister indépendamment des deux autres. C'est ce que nous appelons, faute de mieux, la grammaire intériorisée d'une langue, qui est postulée au cœur de l'objet, quel qu'en soit l'empan, que se donne le grammairien ou le linguiste. L'étude de cet objet implique inévitablement un certain point de vue quant à cette grammaire intériorisée, qui oriente ce que nous appelons une théorie grammaticale. L'artefact qui résulte de l'application d'une de ces théories, ou d'un mixte d'entre elles, à cet objet, est appelé représentation grammaticale (savante).

Certains (pré)conçoivent, en effet, la grammaire comme plutôt tributaire de la logique (adeptes de l'Analogie « tombée du ciel » dans l'Antiquité, Grammaire générale de PortRoyal ou de N. Beauzée, "linguistique cartésienne » de N. Chomsky). D'autres la (pré)conçoivent comme davantage liée à de la rhétorique (Quintilien n'y voyant que l'établissement d'un usage faisant consensus chez les érudits et les honnêtes gens, $\mathrm{Cl}$. Fabre de Vaugelas se livrant à des " perquisitions extraordinaires » afin de décider du " bon \& bel Vsage »,W. Labov fondant la communauté linguistique non sur la diversité des normes objectivement pratiquées mais sur les normes subjectivement reconnues par tous, y compris et surtout par ceux qui ne les pratiquent pas spontanément). Deux points de vue dont l'un fait de la grammaire plutôt une science de l'esprit et l'autre plutôt une science de la société, qui sont liés à l'évolution épistémique du savoir grammatical ou linguistique et qu'il faut croiser avec d'autres développés dans ses sciences « connexes » (Saussure).

Savoir savant, ou d'origine savante, dont les « règles » ont pu être assimilées, au cours de l'histoire et encore parfois, soit aux lois juridiques (moins à celles du droit naturel qu'à celles du droit coutumier, comme c'est le cas chez Vaugelas ou les tenants actuels $\mathrm{du}$ " bon usage »), soit aux « lois de la nature » telles qu'on les a conçues à partir du XVIIe siècle (conception qui perdure chez certains structuralistes adeptes de l'immanence des "structures ", voire chez certains cognitivistes actuels), soit encore être interprétées par certains, dont l'auteur de ces lignes, selon le " nouvel esprit scientifique " dont parlait Bachelard dans les années trente du XXe siècle, interprétation qui en fait des " modèles " à même de reproduire en pensée le réel observé sans que cette représentation mentale coïncide pour autant avec lui (la science « simule » le réel plus qu'elle ne le " découvre »).

Quelle que soit la (pré)conception reçue ou adoptée, la règle du grammairien ou du linguiste suppose, pour qu'elle soit enseignée/apprise à des élèves débutant l'étude d'une L2, une certaine «transposition didactique ». Notion qui ne peut être entendue, 
en didactique des L2, comme elle peut l'être en didactique des sciences physiques et des mathématiques, c'est-à-dire comme la transformation d'un savoir savant en savoir enseignable à des non-spécialistes. La didactique de la linguistique ou de la grammaire (en tant que savoir savant relatif à une langue) peut relever de ce type de " transposition didactique ». Mais une classe de L2 n'est pas une classe de grammaire, et encore moins un cours de linguistique. Pour être enseignée/apprise, c'est-à-dire pour faire en sorte qu'un apprenant qui pratique déjà une ou plusieurs langues parvienne à la parler-écrire plus ou moins comme ceux qui la maîtrisent en tant que L1, une L2 est inévitablement réduite à ce qui en est considéré, dans la tradition scolaire où l'on s'inscrit, comme enseignable. Ce qui revient, le plus souvent, à en réduire la diversité des pratiques sociales à ce qui en a été séculairement traité à cette fin. En d'autres termes, il s'agit moins d'une transposition " descendante ", celle d'un savoir savant qu'il s'agit de transmettre à des non-savants, que d'une transposition " ascendante ", qui fait de simples pratiques sociales, maîtrisées même par ceux qui n'ont pas étudié, quelque chose de transmissible scolairement.

D'où deux traditions dans l'utilisation du savoir des grammairiens et des linguistes dans l'enseignement des L2. L'une, plus « lettrée ", qui s'efforce de le «transposer » au sens que les didacticiens des mathématiques ou de la physique prêtent à ce terme, en présupposant que le savoir "scientifique » relatif aux langues est seul à même de faire progresser la didactique de celles-ci en tant que L2. Du Marsais nous parait s'inscrire dans cette tradition quand il interprète la suggestion de Locke à la lumière de la Grammaire générale ; de même que la " linguistique appliquée » telle qu'elle a pu se développer aux États-Unis autour de 1950. L'autre, plus pédagogique, qui s'efforce d'abord de " transposer " les pratiques sociales constitutives du parler-écrire une langue pour les rendre enseignables, en ne retenant du savoir des grammairiens et des linguistes que ce qui en est plus ou moins passé, au cours des siècles, dans ses pratiques surveillées ou cultivées. C'est dans cette seconde tradition que nous nous inscrivons, en avançant la notion de " culture grammaticale du français ».

\subsection{Sur les techniques d'enseignement de la « grammaire » d'une L2}

A la lumière des propositions qui précèdent, nous caractérisons ensuite les différentes techniques d'enseignement de la grammaire d'une L2, telles qu'elles sont préconisées dans le discours des méthodes ou telles qu'elles sont exemplifiées dans celui des manuels, tant anciens qu'actuels.

En approche bilingue, l'enseignement des règles, en L1 plus qu'en L2, peut précéder (grammaire déductive) ou suivre (grammaire inductive), de façon plus ou moins différée, une certaine pratique de la L2, ce qui a entrainé nombre de débats, de Comenius à nos jours.

126 En approche monolingue stricte, que ce soit en méthode directe ou en méthode S.G.A.V, on est contraint, au moins au départ, à s'en tenir à des techniques inductives, qui ne sont pas incompatibles, au moins dans l'esprit de leurs promoteurs, avec une certaine réflexion grammaticale, menée soit en L1 soit en L2, et pouvant porter, contrastivement, sur ces deux langues, telle celle que nous avons proposée sous le nom de « exercices de conceptualisation ».

127 L'option "grammaire implicite vs. explicite ", telle que précisée par Galisson \& Coste (1976) ou par Stern (1983 et 1992) nous paraît entretenir, dans les discours didactiques, 
confusions et débats en raison de l'ambiguïté de son premier terme, la notion relativement récente de " grammaire implicite ». Elle y renvoie en effet, tout comme grammar dans certains énoncés chomskyens, soit à la grammaire intériorisée d'une langue, soit à une représentation d'origine savante de celle-ci. Et l'on fait ou bien comme si cette représentation coïncidait avec la grammaire intériorisée de la L2, cherchant à en inculquer les règles par la seule pratique de paradigmes d'exemples censés les illustrer, sans qu'on ait à expliciter ces règles en quelque façon (comme dans l'exercice structural tel qu'il s'est répandu en France avant 1970). Ou bien, comme si les élèves étaient à même de " découvrir » ces règles, sans avoir été au préalable instruits des outils théoriques permettant de les (re)construire en tant qu'artefacts (comme dans certaines mises en œuvre de l'Awareness of language). Ou bien encore, comme s'il était possible de reproduire, par diverses techniques de simulation, certaines des conditions qui déterminent l'acquisition « naturelle » des langues, afin que les élèves parviennent à s'approprier, pour ainsi dire, directement la grammaire intériorisée de la L2, sans avoir recours à une quelconque représentation grammaticale savante de celle-ci, ce à quoi tendent nombre de propositions se réclamant de la méthode communicative. Mieux vaudrait sans doute parler dans un cas de grammaire implicitée (" pliée dans » les exemples), et dans l'autre de grammaire implicite, même si cette expression demeure ambiguë.

\subsection{Des (pré)conceptions grammaticales}

Après avoir dressé un récapitulatif des techniques d'enseignement de la " grammaire " d'une L2, nous distinguons trois (pré)conceptions de la règle grammaticale.

L'une est dite juridique, parce qu'elle assimile les règles grammaticales à celles du droit, qu'il s'agisse de ce qu'on qualifie, dans la tradition occidentale, de "droit naturel ", supposé voulu par Dieu ou réputé fondé en "nature ", et donc posé comme existant indépendamment des décisions humaines, ou qu'il s'agisse du « droit coutumier », tel qu'il peut être empiriquement édicté dans chaque société. Les tenants de la Grammaire générale (ceux qui suivent Port-Royal ou Beauzée) privilégient quelque chose qui est de l'ordre du droit naturel ; les tenants des « remarques » grammaticales (ceux qui suivent Vaugelas ou Locke) privilégient quelque chose qui est de l'ordre du droit coutumier. Les uns veulent que la raison précède la routine, et préfèrent donc les techniques déductives ; les autres estiment qu'une certaine routine doit préparer les élèves aux réflexions, et recommandent donc des techniques inductives dont l'explicitation des règles est largement différée. Et selon que l'on est un tenant d'une conception " naturelle » ou " générale » des règles grammaticales, ou un tenant de leur caractère " particulier » ou " arbitraire », on peut distinguer, dans cette (pré)conception juridique deux courants, l'un assimilable au droit naturel, l'autre au droit coutumier.

130 La seconde est dite descriptiviste, parce qu'elle assimile la règle grammaticale à une « loi de (la) nature ", telle que cette notion s'est imposée d'abord aux sciences de la matière, avec Fr. Bacon et R. Descartes, ensuite aux sciences de l'esprit et des sociétés, dont la grammaire. C'est cette (pré)conception, parce qu'elle présuppose l'existence de la règle grammaticale dans la nature même des langues (plus que du langage), qui nous paraît sous-tendre toutes les techniques inductives, avec explicitation non différée de la règle ou sans explicitation de celle-ci, de la " méthode pré notionnelle » d'un P.-A. Lemare aux exercices structuraux tels que conçus par Réquédat [1966] en passant par toutes les 
démarches qui visent à faire en sorte que les élèves " découvrent " par eux-mêmes la règle. Cette (préconception, encore largement dominante en France, impose au mot grammaire, ou à ses équivalents, l'acception inédite jusqu'au XIXe siècle de " grammaire naturelle », que Stern qualifie, à juste titre nous semble-t-il, d'expression « attrape tout ».

La troisième est dite constructiviste, parce qu'elle récuse cette assimilation des règles du grammairien ou du linguiste aux « lois de (la) nature » pour en faire des propositions d'ordre hypothético-déductif visant à rendre rationnellement compte de ce que peuvent être les fonctionnements " grammaticaux » d'une langue, mais propositions qui ne coïncident pas avec ce que sont effectivement ces fonctionnements chez ceux qui pratiquent, nativement ou non, cette langue. Elle correspond au point de vue adopté dans cette étude, qui nous parait, à condition de ne pas l'interpréter comme un relativisme ou conventionnalisme radical, conforme à l'épistémologie des sciences telle qu'elle s'est imposée au cours du XXe siècle.

\section{Chapitre 5 : Comment présenter la langue étrangère}

Les réponses qu'a apportées la didactique occidentale des L2 à notre troisième question mettent en jeu du textuel (que nous limitons au verbal, qu'il soit d'ordre scriptural ou oral) et du discursif (par quoi nous entendons le non verbal au sein duquel un texte est inévitablement communiqué), et qui reviennent toutes à rendre plus ou méthodiquement enseignable ce qui peut s'acquérir, par ailleurs, sans maître. Partant, ceux qui élaborent ou choisissent ces textes que nous disons de présentation de la L2, sont pris dans ce que Stern qualifie de "dilemme code-communication", selon qu'ils se focalisent sur le textuel à présenter ou sur le discursif à même d'assurer sa transmission. L'approche bilingue, en raison des commodités que lui offre la traduction magistrale, tend à focaliser la présentation de la L2 sur le verbal ou le code, alors que l'approche monolingue, quelle que soit la méthode, ne peut éviter le non verbal sans quoi ce code étranger reste hermétique aux élèves.

\section{1. Éléments pour une analyse des textes de présentation d'une $L 2$}

Il s'agit un inventaire - qui ne tient guère compte des méthodes dont ils sont censés relever - des textes ou discours utilisés pour présenter une L2 à des débutants afin de leur en faciliter l'apprentissage. Une première distinction oppose ceux qui sont élaborés directement à cette fin, par les auteurs de manuels (" dialogues didactiques », par exemple) à ceux que ces auteurs extraient à cette même fin des textes et discours ayant été réellement échangés entre des natifs de la L2 (« morceaux choisis » ou « documents authentiques »). Opposition qui pour être simple n'en a pas moins suscité, au cours de l'histoire de l'enseignement des L2, disputes et débats.

134 Sont d'abord examinés différents textes de présentation du français produits pour l'enseigner en tant que L2. Certains apparaissent comme typiques du discours des manuels de L2, en ce qu'ils ne sont qu'une sorte de projection sur l'axe syntagmatique des paradigmes, en particulier grammaticaux, que l'on cherche à inculquer aux élèves. Nous les appelons suite de phrases-exemples, bien qu'ils maintiennent entre les phrases qui les constituent une sorte de cohésion et cohérence. D'autres, les plus nombreux, sont des imitations plus ou moins stéréotypées des discours échangés par les natifs dans 
leurs échanges ordinaires (dialogues, récits ou narrations, plus rarement descriptions), imitations qui visent à introduire, de manière contrôlée et progressive, le vocabulaire et la grammaire de la L2. Ce qui revient souvent, pour reprendre une formulation de H.G. Ollendorff, à " réunir dans un cadre toutes les conversations de la vie, et (à) les adapter aux règles de la grammaire ».

Il nous semble que la figure de Mentor - telle qu'elle est mise en scène par Fénelon dans son Télémaque, ou telle qu'elle est assumée par l'anonyme promoteur de la " méthode Mentor » - est exemplaire de ce genre de textes, en quoi nous voyons un avatar scolaire de ce que l'ancienne rhétorique entendait par " genre didactique ». Genre qui vise à instruire ou à transmettre un certain savoir sous une forme qui, pour ce qui est des manuels de L2, n'est certes pas toujours agréable ou poétique, mais qui présente l'avantage, au moins quant à sa discursivité, d'être familière aux élèves. Et genre dans lequel les auteurs de manuels ne réussissent pas toujours, parce qu'ils y sont pris dans une triple contrainte : a) destinant ces textes à des élèves qui ignorent à peu près tout de la langue qui les tisse et de la culture qui les sous-tend, ils sont plus ou moins contraints de spéculer sur un pré-construit culturel qui soit suffisamment général, et donc relativement banal, afin de ne pas trop les dérouter ; b) les formulations qu'ils adoptent doivent tenir compte de celles qu'ils ont déjà été utilisées dans les unités antérieures, afin que le « nouveau » qui s'y trouve introduit ne soit pas trop lourd à apprendre et n'improuve pas trop les règles antérieurement enseignées : c) il leur faut enfin veiller à équilibrer l'inévitable artifice de ce genre didactique avec les normes ordinaires du type de discours qu'ils privilégient (dialogue, récit, description).

Sont examinés ensuite les textes didactiquement choisis, parmi ceux qui ont été échangés entre des natifs de la L2, pour présenter cette langue à des débutants. Peu présents, au moins à ce niveau, dans les manuels de langue vivante du XIXe et XXe siècles, ils le sont davantage dans les manuels de latin du XVIIe et XVIIIe siècles, quand il fut devenu clair que nul n'écrivait plus et ne parlait plus cette langue comme au temps de Cicéron. D'où le recours à des "modèles de latinité, tirés des meilleurs écrivains latins » (qui ne sont que de simples recueils de phrases "détachées " thématiquement liées) ou à des " endroits choisis " extraits de " quelque auteur facile ", dont Ch. Rollin [1726-1728] conseillait de remanier certaines formulations afin qu'elles soient conformes aux règles de la grammaire, alors que Du Marsais (1722) leur imposait la Construction afin d'en faciliter l'accès aux débutants. Deux transpositions didactiques visant à ce que les textes de présentation de la L2 ne contredisent pas trop, là aussi, la grammaire enseignée. A quoi s'opposera l'abbé Pluche (1751), avant tout soucieux de respecter « le texte des Auteurs comme une chose sacrée ». Un auteur de l'Encyclopédie (Faiguet, 1751) défend cependant, une présentation des langues vivantes telles qu'elles sont journellement pratiquées : " travail pour travail, il vaudrait encore mieux étudier quelque langue moderne comme [...] l'anglois, qui est le plus utile \& (le) plus à la mode ", suggérant à cet effet d'utiliser " la Gazette d'Angleterre qu'on trouve à Paris en plusieurs endroits ", parce que roulant «sur des sujets connus d'ailleurs, pour peu qu'on entende une partie, on devine aisément le reste ». Observation qui sera reprise au début des années 1970, d'abord dans l'enseignement «fonctionnel » du français à des publics spécialisés, avant que la vogue des « documents authentiques » ne la généralise à de présumés « nouveaux débutants ».

137 Le débat entre les tenants de ces deux options apparaît sans fin. 
138 Au XVIIIe siècle, il oppose les défenseurs de « la plus pure latinité » à ceux qui préfèrent débuter par des textes latins " composés exprès " ou remaniés didactiquement (Construction faite, périodes coupées, ellipses restituées, exceptions gommées). Pluche s'en prend ainsi à ceux qui ont élaboré des textes de présentation pour débutants (entre autres, Comenius, Cordier, Erasme), mais aussi et surtout à ceux qui, tel Du Marsais, leur « mettent en main des Auteurs dont ils ont renversé la latinité » en la " ramenant à la phrase Françoise ", ce qui revient, selon lui, à leur présenter " un Latin assurément très-faux ». Ce qui est en jeu dans l'argumentation de Pluche, c'est bien entendu la querelle de l'ordre des mots (dans laquelle il est du côté de Ch. Batteux), mais aussi une conception des « premières leçons des langues » qui seraient « l'ouvrage de nos oreilles \& point du tout de nos réflexions ». Pour lui, les langues entrent « toutes dans un seul \& même mécanisme " qui n'a donc pas à être vraiment enseigné, et comme c'est " la société qui est l'école des langues ", mieux vaut fréquenter celle de Versailles que celle de Limoges.

Dans la seconde moitié du XXe siècle, un débat analogue a opposé, dans les années 1960, les partisans de la "présentation dialoguée ", telle qu'elle était mise en œuvre par la méthode S.G.A.V, aux " monostructuralistes » de la méthode audio-orale, qui présentaient la L2 à apprendre "structure » par "structure ». Girard (1966) et Galisson (1969a) prennent parti pour la " présentation dialoguée », qu'ils jugent plus motivante pour les élèves, mieux adaptée à la langue parlée et plus conforme à son usage réel, tout en reconnaissant que rares sont les auteurs de manuels à y vraiment réussir.

Débat plus ou moins repris, au cours des années 1970, entre ceux qui en tiennent toujours pour la "présentation dialoguée " à ceux qui cherchent à promouvoir les " documents authentiques ». Prélevés non plus chez les « auteurs » mais dans les médias (publicité, journaux, radio, télévision), ceux-ci ont d'abord été utilisés aux niveaux avancés avant de l'être avec des quasi débutants, au nom d'une "authenticité " d'autant plus invoquée qu'elle n'était guère interrogée, en particulier quant à la dénégation du didactique qu'elle engage, en un domaine où l'on voit mal comment on pourrait l'éviter.

141 Les textes de présentation d'une L2, qu'ils soient fabriqués ou qu'ils soient choisis à cette fin, nous paraissent ainsi pris entre trois polarités : la polarité des savoirs, surtout grammaticaux, que l'on cherche à transmettre par leur truchement ; celle des usages de la L2 que ces textes sont censés illustrer ; et la polarité pédagogique, celle des classes mêmes où ils sont utilisés. Trois polarités qui nous paraissent singulariser ce " genre didactique » par rapport à celui de la rhétorique classique, et qui permettent d'établir une typologie de ces textes en fonction de la plus ou moins grande proximité qu'ils entretiennent avec chacune d'entre elles.

\subsection{Comment enseigner ce qui s'apprend sans maître}

Le second mouvement de ce chapitre essaie de montrer qu'une L2 ne peut être enseignée/apprise - qu'on y use de textes élaborés ou de textes sélectionnés - sans qu'elle soit transposée didactiquement, d'une manière ou d'une autre, à cet effet.

Une analyse un peu fine des métamorphoses que subit un texte typique de la méthode directe - texte de Schweitzer \& Simonnot [1902] qui apparaît comme beaucoup plus proche de la polarité pédagogique que des deux autres -, selon qu'il est pris dans le discours de cette méthode, dans celui d'un manuel qui l'applique, ou dans celui d'une 
classe qui suit cette méthode, conduit à distinguer, pour un " même " texte de présentation de la L2, ce que nous appelons le texte-exemple, tel qu'il est présent dans le discours des didacticiens qui le préconisent, le texte-canevas (trame possible de ce qui est à enseigner/apprendre dans une unité) tel qu'il apparaît dans un manuel de L2, et le texte-modèle, c'est-à-dire celui auquel les élèves sont réellement confrontés dans la classe et qui est sans doute celui qui «modélise » le plus leur apprentissage.

Distinctions que nous appliquons, dans un premier temps, aux textes " purs » de Pluche, en montrant combien les huit « exercices » qu'il prévoit afin de les enseigner trois étant « l'affaire du maître » et cinq " l'affaire des commençans » - altèrent leur " pureté " originelle, les élèves étant davantage confrontés, dans les classes où ces exercices sont pratiqués, à leur L1 qu'à une L2 qui s'y trouve réduite à un latin pédagogisé. Et dans un second temps, à ces " documents authentiques " qui sont supposés « faire entrer le réel » étranger dans les classes, en montrant que transférer dans une classe de L2 l'intégralité (par reproduction visuelle ou sonore) d'un " document " est loin de suffire à y transférer ce qui faisait originalement son " authenticité ». Et ce, quelle que soit la technique utilisée pour enseigner/apprendre la langue illustrée par ce document : " explication de texte ", traditionnelle ou modernisée, s'appuyant sur la traduction L2->L1 ; «pré-explication » du texte dans la seule L2, en s'appuyant sur "les procédés directs d'interprétation " tels que les préconisaient les tenants de la méthode directe, ou bien en partant de " textes-filtres » ou de "sous-textes ", tels que ceux dont a pu faire usage dans les années 1970, ou encore en adoptant une démarche " thématique »; « lecture globale " s'appuyant sur divers indices (iconiques, textuels, notionnels, énonciatifs) telle qu'elle a été d'abord développée pour des textes spécialisés avant d'être étendue à d'autres ; enfin, une " éducation à la perception interculturelle " qui tente de combler le "dénivellement interprétatif » que provoque inévitablement le transfert d'un " document authentique " dans une classe de L2, éducation qui suppose un " jeu alterné et cumulé d'identification et de distanciation » (J. Berque) de l'élève par rapport à la L2 et à ce que son usage implique culturellement.

De l'examen de ces différentes techniques, il résulte que " l'artificialité » des textes spécialement élaborés pour présenter une L2 à des débutants, " artificiante » si souvent dénoncée par les tenants des textes " purs " ou des "documents authentiques", se retrouve déplacée, chez les utilisateurs de ces derniers, dans le discours par lequel ils sont effectivement enseignés/appris dans les classes. "Artificialité » d'autant plus accentuée que le maître a recours à des notions et des techniques d'origine savante.

Ce qui nous conduit à regrouper ces différentes démarches dans deux approches - qui ne sont pas sans rapport avec nos approches bilingue vs. monolingue -, l'une que nous disons lettrée ou applicationniste, en ce qu'elle recourt à des outils conceptuels empruntés aux grammairiens ou aux linguistes (de l'eruditio médiévale à la « lecture globale »), l'autre que nous disons pédagogique (des « procédés » de la méthode directe à ceux de « l'éducation à la perception interculturelle »).

147 L'approche "pédagogique " repose sur la conviction que si les infans apprennent à parler leur L1 comme elle se parle, c'est que quelqu'un a pris la peine de la leur « enseigner " quotidiennement. Ce qui n'est qu'un cas particulier de ce que certains psycholinguistes appellent la communication inégale ou dissymétrique, c'est-à-dire entre quelqu'un qui sait, le fort, et quelqu'un qui ne sait pas ou moins, le faible. En matière de langues, au moins deux manières de faire peuvent être adoptées par le fort. Soit le fort 
essaie d'adapter son dire au dire mal maîtrisé du faible, afin d'en être mieux compris ou dans l'illusion de l'être. Il tend alors à adopter, plus ou moins consciemment, un parler simplifié, du type "parler bébé " ou "parler petit nègre ", qui assure certes un minimum de communication, mais qui ne permet guère au faible de progresser dans sa maitrise du parler ordinaire du fort (L. Bloomfield voyait dans cette pratique l'origine des pidgins). Soit le fort, estimant que cette manière de faire est infantilisante voire vulgaire, parle au faible plus ou moins comme il est accoutumé à le faire avec ceux qui maîtrisent sa langue, le contraignant ainsi à s'adapter, sous peine d'être sanctionné s'il n'y parvient pas, au dire du fort. Une voie moyenne est, bien entendu, possible, le fort simplifiant alors son dire sans toucher à la morphologie et la prononciation ordinaire de la langue utilisée. Deux (ou trois) manières de faire qui se retrouvent dans l'enseignement des L2, non seulement entre le maître et ses élèves, mais aussi dans la réflexion didactique portant sur ce type d'enseignement, surtout quand elle tente de fonder « en nature » ses propositions.

Radonvilliers (1768) s'appuie ainsi sur une observation quasi clinique de la manière dont un infans apprend sa L1 auprès de ses proches, afin de montrer que la "méthode » qu'il propose (celle de la " double version ") n'est qu'une "simple imitation de la nature », prédisant que puisque son " art suit exactement les procédés de la nature, il aura le même succès ». Un raisonnement voisin, même s'il n'est pas toujours explicité, nous paraît sous-tendre les recherches actuelles sur " l'acquisition ", non scolaire, des L2. Développées dans le prolongement de l'analyse contrastive et de l'analyse des erreurs, ces recherches postulent que mieux nous comprendrons comment on acquiert une L2 au simple contact de ceux qui la parlent déjà, mieux nous serons à même de les enseigner. Nombre d'entre elles nous paraissent marquées par des présupposés innéistes, ce qui fait que les interactions, au sein desquelles une langue est inévitablement acquise, $\mathrm{n}$ 'y sont guère prises en compte - parfois même par ceux qui en soulignent l'importance, tel Cl. Perdue (1995). Seuls certains " acquisitionnistes " (entre autres M.-T. Vasseur, 1990) redécouvrent l'importance des notions " d'étayage " et de "réglage minutieux », notions que Bruner (1983) a élaborées à partir de multiples observations sur la façon dont les mères « élèvent » leurs bébés. Et encore plus rares sont ceux qui s'intéressent à la description méthodique des manières de faire des natifs " ordinaires » quand ils s'adressent, dans leur L1, à des interlocuteurs adultes ne la maitrisant pas ou mal. Manières de faire qui nous paraissent, pourtant, attester que la prétendue « artificialité » des textes fabriqués présente de fortes ressemblances avec ce que Radonvilliers appelait les " artifices des leçons de la nature ", même si le culturel ou le socio-historiquement situé y a sans doute plus de part qu'il ne le pensait.

La dernière partie du chapitre porte sur la notion de « simplicité » en didactique des L2, telle qu'elle est mise en œuvre dans les textes fabriqués ou sélectionnés pour présenter une L2 à des débutants. On y montre que cette " simplicité » engage plus ou moins les mêmes prérequis que l'étude savante des langues, ceux qui les constituent en objet étudiable et donc enseignable par les sciences du trivium.

150 Comenius [1631] nous paraît, sur ce point, plus explicite que les didacticiens contemporains. Il part d'une critique des pratiques didactiques les plus répandues en son temps, et entreprend de "dresser quelque epitorne de toute la langue, en telle sorte que tous les mots \& toutes les phrases qu'elle a, estans recueillies en un corps, périssent estre apprises en fort peu de temps \& sans beaucoup de travail ». Ce qui serait suivre les « loix de la Didactique naturelle ». Des « loix » qui sont certes liées, comme il 
a été souvent dit, à des observations d'une grande modernité, mais aussi à des considérations théologiques, voulant que la « Didactique naturelle » fasse que chacun riche ou pauvre, garçon ou fille, paysan ou citadin, normal ou " simple d'esprit » puisse recouvrer la « condition première et originelle à laquelle nous devons revenir ». (Pré)conception qui revient à poser qu'une langue (L1 ou L2) doit être enseignée comme on suppose que le langage et les langues l'ont été originellement à l'ensemble de l'espèce humaine. A cette (pré)conception s'en oppose une autre, attestée en particulier chez Blair [1783], qui fait du « style simple » le résultat des " progrès de la civilisation », et qui insiste donc sur son " artifîcialité », au sens valorisant du terme.

Deux (pré)conceptions quelque peu mythiques, auxquelles nous préférons une troisième posant que les notions de « sens littéral » et de "syntaxe simple », inhérentes à celle de "style simple ", sont d'ordre méthodologique. Ce sont des prérequis nécessaires à "l'intelligence " des langues, telle que la tradition occidentale en a développé l'étude plus ou moins scientifique en grammaire, rhétorique et logique. D'où il résulte que l'étude des langues, qu 'elle soit dite savante ou scolaire, demeure une.

\section{Chapitre 6 : Dans quel « ordre » enseigner la L2 ?}

Ce dernier chapitre pose la question de l'« ordre » dans lequel une L2 peut ou doit, selon les méthodes, s'enseigner/apprendre, « ordre » étant entendu à la fois comme ce qui structure l'institution au sein de laquelle cette L2 est enseignée/apprise, et comme ce qui distribue les éléments, linguistiques ou non, constitutifs de cette langue dans la succession des unités d'un manuel. La tradition didactique française a longtemps raisonné cet " ordre » en termes de plan d'études, de programmes (scolaires) ou de cursus, ou en termes de contenu et de progression (d'enseignement ou d'apprentissage). Les sociologues de l'éducation anglo-saxons ont imposé, depuis une trentaine d'années, les termes de curriculum et de syllabus, termes d'origine gréco-latine, mais dont les acceptions actuelles diffèrent beaucoup de celles qu'ils avaient dans les textes latins et grecs.

Plutôt que de tenter, à partir de ce qui a été écrit récemment à ce sujet, une reconceptualisation critique du domaine concerné, nous avons préféré, sans ignorer ces propositions, adopter un point de vue historique s'inscrivant dans la longue durée. Dans une première partie de ce chapitre sont donc rappelées les réflexions par lesquelles on a cherché, en Occident, à raisonner l'ordre curriculaire, d'abord à propos du latin et du grec (en tant que L1 puis en tant que L2), ensuite des langues vulgaires (d'abord en tant que L2). Et dans les seconde et troisième, sont rappelées les réflexions par lesquelles, dans cette même tradition occidentale, on a raisonné l'ordre syllabaire.

\subsection{Quelques jalons pour une " archéologie » de la réflexion curriculaire}

Les trois « cas " que nous avons retenus pour esquisser une " archéologie " de la réflexion curriculaire attestent qu'elle était liée, dès l'Antiquité, à des options différentes quant au fait de savoir s'il valait mieux enseigner plusieurs langues à la fois ou l'une après l'autre, et quant au fait de savoir si leur enseignement devait s'inscrire ou non dans une éducation « encyclopédique ». 
Quintilien (1975) est partisan, à la fin du 1er siècle, d'un enseignement très vite parallèle du grec et du latin (L1 plus que L2), même s'il conseille de débuter par le grec parce que le latin est " plus usité », enseignement assuré par un « maître grec » et par un " maître latin ", l'un et l'autre faisant appel aux mêmes « disciplines »- celles du latin dérivant de celles du grec -, avant que l'élève ne fréquente d'autres maîtres (de musique, de mathématiques et de comédie). Formation qui vise moins à véritablement instruire le futur orateur qu'à l'éduquer en tant que citoyen romain apte à manier une parole publique ayant quelque influence dans les décisions de la Cité. Ce qui requiert une progressive accoutumance à " l'accord des honnêtes gens et des gens cultivés (consensus bonorum et eruditorum) " quant à l'usage des langues utilisées et quant aux savoirs à même de lester le discours d'une «force latente ».

Les jésuites (Ratio studiorum, 1997), à la fin du XVIe siècle, adoptent un dispositif curriculaire voisin, bien qu'il soit marqué par un modus parisiensis dont Quintilien ne pouvait avoir idée. Ils préconisent d'enseigner parallèlement le latin et le grec dès la première classe de "grammaire ", les élèves étant des faux-débutants en latin et des vrais débutants en grec. Enseignement assuré par un même " régent " montant de classe en classe avec ses élèves, que sa formation a rendu apte à instiller, en particulier dans la pratique de la praelectio, des éléments d'eruditio et de morale chrétienne.

Comenius (1992), au début du XVIIe siècle, rompt avec cette tradition curriculaire " classique ", en préconisant, après le père de Scaliger, de n'étudier qu'une langue à la fois, et en adaptant l'enseignement successif des autres langues à « la langue déjà sue ». Dans l'école comménienne - qui demeure encore en partie une utopie - tous les enfants étudient d'abord leur L1 conjointement aux « choses » dont elle permet de parler, en particulier ces « arts mécaniques » où l'homme a si bien imité son Créateur. Puis ils apprennent la ou les " langue(s) du pays voisin ", d'abord en relisant les livres déjà utilisés à l'école élémentaire mais traduits dans cette (ces) L2, ensuite en séjournant dans ce pays. Ceux qui « aspirent à des fonctions plus hautes que les fonctions artisanales " entrent alors à " l'école latine " où ils apprennent le latin à l'aide de manuels adaptés non seulement à leur L1 mais aussi aux savoirs, en particulier grammaticaux, qui leur ont été antérieurement enseignés à propos de cette L1 et de « la langue du pays voisin ». Enfin, ceux qui se destinent à devenir théologiens ou médecins complètent leur formation en étudiant, à partir de ce qui a déjà été appris à propos de la L1 et du latin, le grec et l'hébreu pour les premiers, le grec et l'arabe pour les seconds, moins pour parler ces langues que pour lire les livres écrits dans celles-ci.

La pertinence de cette ancienne réflexion curriculaire, qu'elle soit « classique » ou plus novatrice, sera en grande partie oubliée, quand l'enseignement des " choses " l'emportera sur celui des « mots" dans la seconde moitié du XVIIIe siècle, quand cette réflexion sera confiée au législateur ou à des inspecteurs inscrivant leurs propositions dans des lois et des instructions officielles qui, étant peu ouvertes au débat, ne sont guère problématisées, et quand l'enseignement des L2 vivantes sera très progressivement institutionnalisé au cours du XIXe siècle, sans que soit vraiment posée, en raison d'un compartimentage des disciplines scolaires de plus en plus rigide, la question de l'adaptation de leur enseignement à la « langue déjà sue ».

C'est aussi un point de vue historique, bien que moins " archéologique ", qui oriente notre analyse des réponses que l'on a apportées, en Occident, à la question syllabaire dans l'enseignement des L2, qu'il s'agisse de la sélection de la variété à enseigner et des 
progressions d'enseignement adoptées, ou qu'il s'agisse des groupages destinés à faciliter l'apprentissage.

\subsection{La réflexion syllabaire relative à la sélection et aux progressions}

Après avoir rappelé ce qu'était, pour Vaugelas, le "bon usage ", celui de « l'élite des voix » ou de la sanior pars, et ce qu'étaient les "soins \& perquisitions extraordinaires " au moyen desquels il l'établissait, nous montrons que ce " bon usage » suppose un traitement social de la langue qui engage des savoirs d'ordre juridique, linguistique et esthétique. Traitement qu'on retrouve, mutatis mutandis, dans les analyses d'un W. Labov, en particulier dans ses " normes subjectives », tout comme dans «l'idéologie de la norme " selon Milroy \& Milroy (1985). Traitement que nous paraissent occulter nombre de linguistes du XIXe et XXe siècles, quand ils tentent d'objectiver, en invoquant « le plus grand nombre », ce qu'ils appellent " la norme » ou " le standard » d'une langue. L'étude méthodique d'une langue, qu'elle soit le fait du savant ou d'un élève qui commence à apprendre une L2, nous semble inévitablement normative, et si la variété cultivée est préférée, dans cette étude méthodique, aux variétés vernaculaires, c'est qu'elle est la seule à avoir été socio-historiquement "outillée " pour être ainsi enseignée/apprise.

161 La sélection étant opérée, par nécessité plus que par choix, reste à savoir ce que, dans cette variété cultivée, on enseigne en premier et ce qu'on enseigne conjointement dans une même unité. Reprenant le "triangle didactique » de Legendre - qui nous a déjà servi à répartir les textes de présentation d'une L2 entre trois pôles - nous distinguons dans les progressions d'enseignement (celles d'apprentissage relevant d'une analyse différente), les progressions plutôt centrées sur la matière enseignée (sur " l'objet »), les progressions plutôt centrées sur l'enseignant (sur « l'agent »), ou plus exactement sur les techniques dont il dispose pour faire apprendre une L2 à des débutants, et les progressions plutôt centrées sur l'apprenant (sur le « sujet »). Mais, bien entendu, la centration sur l'un des trois pôles n'exclut pas totalement les deux autres -sauf parfois dans certains discours des méthodes.

Les progressions plutôt centrées sur « l'objet » enseigné sont sans doute les plus anciennes (l'instruction grecque et étrusque en relèveraient selon H.-I. Marrou). Elles sont bien exemplifiées dans les " manuels complexes » du XVIIe et XVIIIe siècles - certains continuant à être réédités jusqu'au début du XXe siècle - ayant le français comme $\mathrm{Ll}$ ou comme L2, entre autres ceux de Veneroni, Des Pepliers, Junker, Chantreau. Suivant plus ou moins « le plan de Quintilien » dans son Liber primus, ils comprennent deux grandes parties. La première, intitulée " grammaire ", traite la matière à enseigner selon les règles de l'ars grammatica, c'est-à-dire en débutant par la prononciation (à entendre comme l'oralisation selon les normes de la L2 d'un alphabet déjà plus ou moins familier aux élèves) et l'orthographe, puis en passant aux parties du discours, traitées, en L1 et en L2, à peu près toujours dans le même ordre, enfin la syntaxe, abordée comparativement, à quoi s'ajoutent parfois quelques éléments de " poétique ». La seconde, dont les titres varient selon les manuels, traite cette même matière dans la tradition des etymologiae et des nominalia. Elle regroupe d'abord les noms et les verbes (plus rarement les adjectifs) réputés « les plus usuels » ou " les plus familiers » sous un certain nombre de thèmes (« Du Ciel \& des Elémens », « Des parties du corps », « Pour l'étude », « Pour boire \& manger »...). Elle présente ensuite deux ou trois dizaines de « 
dialogues familiers » («Pour faire une visite le matin », « Pour acheter » ou « Pour monter à cheval »), dialogues suivis d'un recueil de " manières de parler » regroupées par « actes » (« Pour prier \& exhorter », « Pour nier », « Pour admirer », « Pour appeler $»)$, un peu à la manière des " actes de parole » que l'on trouve dans les inventaires actuels du type «niveau-seuil ». Enfin, cette seconde partie se termine par divers autres recueils (de proverbes, de « coutumes", de lettres. ..). Pour ce qui est de la "grammaire ", nous examinons plus particulièrement l'ordre dans lequel y sont traitées les parties du discours. Pour ce qui est de la "langue ", nous examinons l'ordre dans lequel sont présentés les thèmes des "dialogues familiers " et des " manières de dire ", avant de nous livrer à quelques spéculations sur la manière dont on pouvait utiliser ces manuels dans les classes.

163 Si l'on compare cet ordre d'une part à celui qui ordonne les cent titulis de la Ianua de Comenius, d'autre part à ce qu'il devient dans des manuels du XIXe siècle reprenant ce mode de présentation (ceux de Cobbett, Vergani, Spiers), on ne peut que constater un étiolement de cette tradition, dont les derniers avatars au XXe siècle sont sans doute des recueils du type Les mots anglais et les locutions anglaises groupés d'après le sens de $\mathrm{F}$. Novion.

Les progressions plutôt centrées sur l'enseignant se développent surtout au XIXe siècle. Curieusement, d'abord dans des manuels destinés à l'auto-apprentissage, où il faut préciser à l'élève comment il doit s'enseigner lui-même, tels les manuels de T. Robertson. La méthode « synthétique » de H. G. Ollendorff en est un bon exemple, qui introduit " les phrases de manière que le professeur ait la faculté d'adresser les questions dans la langue même qu'il enseigne, et les élèves celle de lui répondre ". La méthode directe en est un autre exemple, l'enseignant, méthodologiquement privé de l'usage de la L1, devant s'en tenir, avec les débutants, à ce qu'il est à même de leur enseigner « directement » en L2.

Les progressions plutôt centrées sur l'apprenant sont sans doute aussi anciennes que les précédentes (certaines propositions de Quintilien ou de Du Marsais en relèvent), mais c'est surtout dans la seconde moitié du XXe siècle qu'elles ont été revendiquées comme telles, dans les méthodes audio-orale, S.G.A.V, notionnelle-fonctionnelle et communicative. L'« analyse contrastive » qui sous-tend en principe, la méthode audioorale nous paraît pouvoir être classée dans ce type de progressions, dans la mesure où R. Lado, l'un de ses promoteurs, prétend " découvrir tous les problèmes d'apprentissage ", c'est-à-dire prédire et prévenir les difficultés qui seront celles d'un élève qui pratique telle $\mathrm{L} 1$ et qui apprend telle L2. En dépit de ce qui a pu être écrit à ce propos durant les dernières décennies, nous pensons que le point de vue de G. Gougenheim (1962) dans l'élaboration du Français fondamental (1er degré) relève de ce même type de progression. Ne s'agit-il pas d'établir, en ayant recours aux statistiques et pour un adulte " avide de résultats pratiques ", un vocabulaire et une grammaire limités du français parlé, afin d'enseigner à cet adulte "un savoir restreint mais immédiatement utilisable, et, si possible, un savoir en rapport avec ses besoins " ? Raisonnement qui n'est pas très éloigné de celui qui sous-tendra, deux décennies plus tard les travaux du Conseil de l'Europe, qui sont à l'origine du notionnel-fonctionnel, avant que celui-ci ne soit critiqué par un communicatif spéculant sur la possibilité d'établir des progressions centrées sur les activités mêmes d'apprentissage (processoriented), progressions dont nous n'avons pas trouvé d'exemplification dans les manuels actuels de français L2. 


\subsection{La réflexion syllabaire relatives aux groupages} retenus. Nous y contestons maintes propositions actuellement véhiculées par le discours des didacticiens des L2, et nous cherchons à y expliciter, contrairement à ce discours qui les mentionne sans vraiment les démonter, les principales techniques d'enseignement que l'Occident a développées pour enseigner les L2, ainsi qu'une partie des « croyances » qu'elles engagent et que leur mise en œuvre répétée dans les manuels et dans les classes a fini par "naturaliser ». Dans ces analyses, nous nous sommes souvent heurté sinon à l'impensé du moins au peu pensé en linguistique, et qui, pourtant, sous-tend nombre des raisonnements qu'on y suit. D'où, parfois, l'impression de « débroussailler " un terrain peu pratiqué, la présence de certaines notes attestant que d'autres pistes ont été ouvertes puis abandonnées.

Mais ces résultats conduisent aussi à problématiser, davantage qu'il ne l'est, l'enseigner/apprendre les L2. Sans nier l'organisation neuro-biologique qui permet à la seule espèce humaine de développer l'apprentissage des langues, ils montrent que cet apprentissage est, pour ce qui dépend de l'action enseignante, déterminé par un enseigner/apprendre socio-historiquement situé. En d'autres termes, cette thèse 
aboutit à « dénaturaliser » la didactique des L2, ce qui n'est pas sans implication quant aux rapports que cette didactique peut entretenir avec ses sciences connexes - qu'elles soient linguistiques, psychologiques ou cognitives -, et quant à la formation des enseignants de langue(s).

\section{BIBLIOGRAPHIE}

Bibliographie du résumé ${ }^{7}$

ALLWRIGHT, D. (1991) : « The Death of the Method », Revue de Phonétique Appliquée, 99-100-101,79-87.

ANTHONY, E. M. [1963] (1965) : « Approach, method and technique », 93-97 dans : Teaching English as a Second Language ed. par H.B. Allen. New York : Mc-Graw Hill.

BESSE, H. (1985) : Méthodes et pratiques des manuels de langue. Paris : Didier (coll. 'Essais').

BESSE, H. ; PORQUIER, R. (1984) : Grammaires et didactique des langues. Paris : Hatier-Crédif (coll. LAL ).

BIALYSTOK, E. (1981) : « The role of linguistic knowledge in second language use », Studies in Second

Language Acquisition, 4, 31-35.

BIBEAU, G. (1983) : « La théorie du Moniteur de Krashen : aspects critiques », Bulletin de l'ACLA, 5 (1),

99-123.

BLAIR, H. [1783] (1797) : Leçons de rhétorique et de belles-lettres. Par le Docteur Hugh Blair, Professeur de Rhétorique à l'Universitéd'Edimbourg ; traduit de l'Anglais par M. Cantwell. - Paris : chez Gide, $\mathrm{L}$ 'an $\mathrm{V}$, en quatre vols.

Bosco, F.-J.) ; Di PIETRo, R.-J. (1970), « Instructional Strategies : Their Psychological and Linguistic Bases », IRAI, VUJ ( 1 ), $1-19$.

BRUNER, J.-S. (1983) : Le développement de l'enfant : Savoir faire, savoir dire ; traduit et présenté par M. Deleau. Paris : Presses Universitaires de France, 1983.

COMBettes, B. ; LAGARDE, J.-P. (1982) : « Un nouvel esprit grammatical », Pratiques, nº 33, mai, 13-49. COMEnIUS, J. A. [1631] (1643) : ;anua Linguarum Reserata, Cum Greca versione Theodorì Simonii Holsati, Secunda hac editione recognita, \& innumeris in locis emendata : Et Gallica nova Stephani Curcelloei. Amstelodami : Apud Ludovi-cum Elzevirum.

COMENIUS, J.-A. (1992) : La Grande Didactique ou l'art universel de tout enseigner à tous. Traduction M.-F. Bosquet-Frigout, D. Saget, B. Jolibert. - Paris : Editions Klinsieck, 1992.

CosTE, D. (1970) : « Le renouvellement méthodologique dans l'enseignement du français langue étrangère : remarques sur les années 1955-1970 \%, Langue française, 8, 7-23.

COSTE, D. (1985), « Métalangages, activité métalinguistique et enseignement/apprentissage d'une langue étrangère », DRLAV, 32, 63-92.

DABENE, M. (1972) : « Didactique des langues et disciplines fondamentales », Voix et Images du Crèdif $15,1972,14-15$. 
DEBYSER, F. (1973) : « La mort du manuel ou le déclin de l'illusion méthodologique », Le français dans le monde, 100, 63-68.

DUBOIS, J. et autres (1973) : Dictionnaire de linguistique. Paris : Librairie Larousse.

DUBOIS, J. et autres (1994) : Dictionnaire de linguistique et des sciences du langage. Paris : Larousse.

Du MARSAIS, C. Chesneau (1722) : Exposition d'une méthode raisonnes pour apprendre la langue latine. Suivie de Le Poème séculaire d'Horace. A. Paris, E. Ganeau.

FAIGUET, F. (1751) : art. « Etude », vol. 6, 89-96. dans Encyclopédie ou Dictionnaire raisonné des sciences, des arts et des métiers. Nouvelle impression en fac-similé de la première édition de 1751-1780. Stuttgart - Bad Cannstatt : Friedrich Fromman Verlag (Günter Holzboog), 1966-1967.

FOERSTER, C. (1993) : « Du côté des représentations et attitudes de l'apprenant : les dessous de la grammaire en langue maternelle et étrangère », LID1L, 9, déc, 11-33.

GALISSON, R. (1969a) : « Le dialogue dans l'apprentissage d'une langue étrangère », Le français dans le monde, 63, mars, 6-11.

GALISSON, R. (1969b) : Petit lexique d'initiation à la linguistique appliquée et à la méthodologie. Paris : Bureau pour l'Enseignement de la Langue et de la Civilisation (BELC)/Faculté des lettres et sciences humaines de Besançon (multigr.).

GALISSON, R ; COSTE, D. dir. (1976) : Dictionnaire de didactique des langues. Paris : Hachette.

GERMAIN, C. (1989) : Les grands courants en didactique des langues secondes Montréal : copyright Claude Germain (multigr.).

GERMAIN, C. (1993) : Evolution de l'enseignement des langues : 5000 ans d'histoire. Paris : CLE International (coll. 'Didactique des langues étrangères').

GIORDAN, A. ; VECCHI, G. de (1987) : Les origines du savoir. Des conceptions des apprenants aux concepts scientifiques. Neuchâtel/paris : Delachaux \& Niestlé (coll. 'Actualités pédagogiques et psychologiques').

GIRARD, D. (1966) : « Pour ou contre le dialogue », Le français dans le monde,38,12-20.

GOUGENHEIM, G. (1962) : « Principes nouveaux pour l'enseignement du français », Esprit. Numéro spécial « Le français langue vivante », n 11, novembre, 585-594.

GREGG, K. R. (1984) : « Krashen's Monitor and Occam's Razor », Applied Linguistics, 5 (2), 79-100.

HALLIDAY, M. A. K. ; MCINTOSH, A. ; STREVENS, P. (1964) : The Linguistics Sciences and Language Teaching. London : Longman.

HAMMERLY, H. (1985) : An integrated Theory of Language Teaching. And its practical consequences. Blaine, WA : Second Language Publications.

HAYNE, J.-M. ; YORIO, C.-A. (1982) : « Adult views on foreign language teaching methods ", 145-149, dans P. R. LEON, J. YASHINSKY (dir.) : Options nouvelles en didactique du français langue étrangère. Montréal : Didier.

JAKOBSON, R. (1963) : Essais de linguistique générale ; traduit de l'anglais et préfacé par Nicolas Ruwet. Paris : Les Editions de Minuit (coll. 'Arguments').

JOLIBERT, B. (1987) : Raison et éducation. Paris : Editions Klincksieck (coll. 'Philosophie de l'Education').

KELLY, L.-G. [1969] (1976) : 25 Centuries of Language Teaching. Rowley, Mass. : Newbury House Pub. 
KRASHEN, S. D. (1981) : Second Language Acquisition and Second Language Learning. Oxford : Pergamon Press.

KRASHEN, S. D. (1989) : Language Acquisition and Language Education. New Jersey/London : Prentice Hall International.

KRASHEN, S. D. ; SELIGER, H. W. (1975) : « The Essential Contributions of Formal Instruction in Adult Second Language Learning », Tesol Quartely, 9 (2), 173-183.

KRASHEN, S. D. : TERRELL, T. D. (1983) : The Natural Approach : Language Acquisition in the Classroom. Oxford/San Francisco : Pergamon/Alemany.

KUANG-JANE, Y. (1992) : «L'apprenant chinois face au métalangage grammatical », LIDIL, 5, février, 109-123.

LARSEN-FREEMAN, D. (1986) : Techniques and Principles in Language Teaching. New York, Oxford University Press.

LEGENDRE, R. (1988) : Dictionnaire actuel de l'éducation. Paris/Montréal : Larousse.

MACKEY, W.-F. [1965] (1972) : Principes de didactique analytique. Analyse scientifique de l'enseignement des langues. Nouvelle édition traduite et mise à jour par L. Laforge avec la collaboration de l'auteur. Paris : Didier.

MARTON, W. (1988) : Methods in English Language Teaching : Frameworks and Options. New Jersey : Prentice Hall International.

MCLAughlin, B. (1978) : « The Monitor Model : Some methodological considerations ». Language Learning, 22 (2), 309-332.

MCLAUGHLIN, B. (1987) : Theories of Second-Language Learning. London : Edward Arnold.

MILNER, J.-C. (1989) : Introduction à une science du langage. Paris : Seuil (coll. 'Des travaux').

MILROY, J. ; MILROY, L. (1985) : Authority in Language. London : Routledge \& Kegan Paul.

MOUNTN, G. dir. (1974) : Dictionnaire de la linguistique. Paris : Presses Universitaires de France.

ODLIN, T. (1986) : « On the Nature and Use of Explicit Knowledge », IRAL, XX/V (2), 123-144.

PERDUE, C. (1995) : L'Acquisition du français et de l'anglais par des adultes. Former des énoncés. Paris : CNRS Editions.

PLUCHE, N.-A. (1751) : La mécanique des langues, ou l'art de les enseigner. Paris : Veuve Estienne \& Fils.

POTTIER, B. et al. (1973) : Le Langage. Paris : Centre d'Etude et de Promotion de la Lecture ('Les dictionnaires du savoir moderne').

PUREN, C. (1988) : Histoire des méthodologies de l'enseignement des langues. Paris : Nathan/CLE International (coll. 'Didactique des Langues Etrangères').

QUINTILIEN (1975) : Institution oratoire. Texte établi et traduit par Jean Cousin. -Paris : « Les belles lettres », en 2 vols. ('Collection des universités de France').

[RADONVILLIERS, C. F. Lizarde de] (1768) : De la manière d'apprendre les langues. Paris : Saillant.

Ratio studiorum.

Plan raisonné et institution des études dans la Compagnie de Jésus. (1997). Édition bilingue latinfrançais. Présentée par A. Demoustier et D. Julia, traduite par L. Albrieux et D. Pralon-Julia, annotée et commentée par M.-M. Compère. Paris : Belin. 
REQUEDAT, Fr. [1966] (1973) : Les exercices structuraux. Seconde édition. Paris :Hachette/Larousse,. RICHARDS, J.-C. ; RODGERS, T.-S. (1986) : Approaches and Methods in Language Teaching. A description and analysis. Cambridge, London, New York : Cambridge University Press.

RIVERS,W. [1968] (1972) : Teaching Foreign-Language Skills. Chicago and London : University of Chicago Press.

ROLLIN, Ch. [1726-1728] (1732) : De la maniere d'enseigner et d'étudier les belles lettres, par rapport à l'esprit \& au coeur. Quatrième Édition, revue, corrigée, \& augmentée d'une Table des matières. Quatre volumes. Paris : Jacques Estienne.

SCHWEITZER, Ch. ; SMONNOT, E. [1902] (1921) : Méthodologie des langues vivantes. [2 éd., « refonte et extension d'une brochure parue sous le même titre en 1902 »]. Paris : Armand Colin.

STERN, H. H. (1983) : Fundamental Concepts of Language Teaching. Oxford : Oxford University Press.

STERN, H. H. (1992) : Issues and Options in Language Teaching. Ed. by Patrick Allen and Birgit Harley. Oxford : Oxford University Press.

VASSEUR, M.-T. (1990) : « La communication entre étrangers et autochtones : stratégies pour se comprendre, stratégies pour apprendre », 239-260, dans La communication inégale, dir. par F. François.- Neuchâtel/Paris : Delachaux et Niestlé (coll. 'Actualités pédagogiques et psychologiques').

VAUGELAS, Cl. Fabre de [1647] (1934): Remarques sur la Langue Françoise. Facsimile de l'édition originale. Paris : E. Droz.

ZIMMERMANN, G., en coll. avec E. WISSNER-KURKAWA (1984) : Erkundungen zur Praxis des Grammatikunterrichts. Frankfurt am Main : Diesterweg.

\section{NOTES}

1. Depuis 1985, il n'est plus possible, en France, de s'inscrire à un doctorat d'État, mais ceux qui y étaient inscrits à cette date ont encore la possibilité d'en soutenir un.

2. Elle comprend environ mille cinq cents pages réparties en quatre tomes. Les trois premiers, d'environ quatre à cinq cents pages chacun, constituent le corps de l'étude, chacun s'ouvrant sur la table des matières des deux chapitres qu'il renferme et se terminant par les index des noms propres et des principales notions qui y apparaissent. Quant au quatrième tome, il comprend la conclusion générale de l'étude, ses annexes et ses bibliographies. Cette thèse ne sera pas publiée telle quelle, mais elle a été reproduite en vingt-quatre exemplaires destinés aux membres du jury, à quelques bibliothèques universitaires (École normale supérieure Lettres \& Sciences humaines implantée à Lyon ; Universités de Paris VII et de Paris VIII ; Institut de Ciències de l'Educació de l'Universität Autònoma de Barcelona...), ou aux chercheurs qui souhaitent en disposer (s'adresser à l'auteur).

3. Des chercheurs occidentaux ont interrogé leur propre tradition philosophique ou scientifique à partir, par exemple, de la tradition chinoise, mais il n'existe, à notre connaissance, que peu de recherches adoptant ce point de vue en didactique des L2.

4. Les crochets renvoient à la date de la première édition de l'ouvrage ou de l'article référencé, quand nous le citons dans une traduction ou édition ultérieure dont on trouvera la date en bibliographie

5. Les termes en italiques sont ceux que nous avons retenus dans la suite de notre travail. 
6. Les dénominations des méthodes sont en italiques quand elles apparaissent pour la première fois dans notre résumé.

7. La bibliographie des seules références de la thèse comprend près de mille titres. Nous en tenons à ceux qui nous ont semblé indispensables à la compréhension de ce résumé.

\section{AUTEUR}

\section{HENRI BESSE}

120-168 